\title{
Boundary measures, generalized Gauss-Green formulas, and mean value property in metric measure spaces
}

\author{
Niko Marola, Michele Miranda jr. and Nageswari Shanmugalingam
}

\begin{abstract}
We study mean value properties of harmonic functions in metric measure spaces. The metric measure spaces we consider have a doubling measure and support a (1,1)-Poincaré inequality. The notion of harmonicity is based on the Dirichlet form defined in terms of a Cheeger differentiable structure. By studying fine properties of the Green function on balls, we characterize harmonic functions in terms of a mean value property. As a consequence, we obtain a detailed description of Poisson kernels. We shall also obtain a Gauss-Green type formula for sets of finite perimeter which posses a Minkowski content characterization of the perimeter. For the Gauss-Green formula we introduce a suitable notion of the interior normal trace of a regular ball.
\end{abstract}

\section{Introduction}

Solving the Dirichlet problem on a smooth domain in $\mathbb{R}^{n}$ is equivalent to constructing harmonic measure on the boundary of the domain. More precisely, it is known that the classical harmonic measure can be expressed in terms of a Poisson kernel which is given by the Radon-Nikodym derivative of harmonic measure with respect to the Hausdorff boundary measure; that is,

$$
P(x, y)=\frac{d \nu_{x}}{d \mathcal{H}^{n-1}}(y)
$$

In general metric measure spaces with a doubling measure and a Poincaré inequality, the Dirichlet problem has been solved for Sobolev type boundary data in [24], and also for all continuous boundary values in [4]. In [4] the authors provide an integral representation for the solution to the Dirichlet problem, and

Mathematics Subject Classification (2010): Primary 31C35; Secondary 31C45, 30C65.

Keywords: Dirichlet form, doubling measure, functions of bounded variation, Gauss-Green theorem, Green function, harmonic function, metric space, Minkowski content, Newtonian space, perimeter measure, Poincaré inequality, singular function. 
hence extend the solvability to $L^{1}$ boundary data. In this general setting, however, due to lack of a natural choice of boundary Hausdorff measure one has to replace a Poisson kernel with a Poisson kernel-like object for which

$$
P_{x_{0}}(x, y)=\frac{d \nu_{x}}{d \nu_{x_{0}}}(y)
$$

It was shown in [4] that for a fixed $x_{0} \in \Omega$, where $\Omega$ is a bounded open subset of $X$, there exists a Radon measure $\nu_{x_{0}}$ concentrated on $\partial \Omega$, i.e., $\nu_{x_{0}}$ is a harmonic measure on $\partial \Omega$ evaluated at $x_{0}$, and a real-valued function $P_{x_{0}}$ on $\Omega \times \partial \Omega$ such that whenever $f \in L^{1}\left(\partial \Omega, \nu_{x_{0}}\right)$ the following expression for the harmonic extension $H_{f}$ is valid:

$$
H_{f}(x)=\int_{\partial \Omega} f(y) P_{x_{0}}(x, y) d \nu_{x_{0}}(y)
$$

and moreover, for each $y \in \partial \Omega$ the function $P_{x_{0}}(\cdot, y)$ is harmonic in $\Omega$.

Our main objective is to find a relationship between the Poisson kernel that generates solutions to the Dirichlet problem in terms of Cheeger differentiable structure, and the perimeter measure of a ball of finite perimeter in metric measure spaces. Our framework is a complete geodesic metric measure space with a doubling Borel measure, and we moreover assume that the space supports a $(1,1)$ Poincaré inequality. These conditions are discussed in detail in Section 2. We shall describe the Poisson kernel in terms of an analog of a normal derivative of the Green function at the boundary.

We also study divergence-measure fields along the lines of Ziemer [27] in this general context. We consider an $L^{2}$-vector field, $\vec{F}$, from a metric measure space $X$ to $\mathbb{R}^{k}$ for which $\operatorname{div} \vec{F}$ is a real-valued signed Borel measure with finite mass.

To investigate divergence-measure fields we shall provide a meaningful definition for the divergence operator in metric measure spaces. We then generalize some results obtained in [27] to the metric setting. In particular, we obtain the Gauss-Green type integration by parts formula for sets of finite perimeter which possess a Minkowski content characterization of the perimeter. For the GaussGreen formula we introduce a suitable notion of the interior normal trace of a regular ball.

We mention a related paper by Thompson and Thompson [26] in which the authors define divergence and prove an analogue of the Gauss-Green theorem in Minkowski spaces, i.e., in finite-dimensional real normed spaces with smooth and strictly convex unit ball.

We use the results for the divergence operator to characterize the Laplace operator of the Green function on regular balls as the sum of the Dirac point mass and a measure concentrated on the boundary of the ball. This characterization allows us to give a precise description of the Poisson kernel defined in [4]. In the setting of Heisenberg groups, we explain the relation between this measure and the perimeter measure or the codimension one Hausdorff measure. 


\section{Preliminaries}

Here we recall some basic definitions and the notation we shall use in this paper. Our framework is given by a complete metric measure space $(X, d, \mu)$, where $\mu$ is doubling, that is, there is a constant $c>0$ such that for every ball $B=B_{r}(x)$, $x \in X$ and $r>0$,

$$
0<\mu(2 B) \leq c \mu(B)<\infty .
$$

We write $B_{r}(x)$ for the ball centered at $x$ with radius $r>0$, and $\lambda B=B_{\lambda r}(x)$ for any $\lambda>0$. The smallest value of $c$ for which (2.1) is valid is called the doubling constant of $X$, and we shall denote it as $c_{d}$.

An upper gradient for an extended real-valued function $u: X \rightarrow[-\infty,+\infty]$ is a Borel function $g: X \rightarrow[0, \infty]$ such that

$$
\left|u(\gamma(0))-u\left(\gamma\left(l_{\gamma}\right)\right)\right| \leq \int_{\gamma} g d s
$$

for every nonconstant compact rectifiable curve $\gamma:\left[0, l_{\gamma}\right] \rightarrow X$. We say that $g$ is a $p$-weak upper gradient of $u$ if (2.2) holds for $p$-almost every curve; the notion of $p$-almost every curve is in the sense of the $p$-modulus of a curve family $\Gamma$ defined as

$$
\operatorname{Mod}_{p}(\Gamma)=\inf \left\{\int_{X} \varrho^{p} d \mu: \varrho \geq 0 \text { is a Borel function, } \int_{\gamma} \varrho d s \geq 1 \text { for all } \gamma \in \Gamma\right\} .
$$

If $u$ has an upper gradient in $L^{p}(X, \mu)$, then it is possible to prove the existence of a unique minimal $p$-weak upper gradient $g_{u} \in L^{p}(X, \mu)$ of $u$, where $g_{u} \leq g \mu$-a.e. for every $p$-weak upper gradient $g \in L^{p}(X, \mu)$ of $u$. We refer to [24] for the case $p>1$, and for the case $p=1$ to [12].

In what follows, the metric space is supposed to support a weak $(1,1)$-Poincaré inequality: there exist constants $c>0$ and $\lambda \geq 1$ such that for all balls $B_{r}$ with $B_{\lambda r} \subset X$, for any Lipschitz function $f \in \operatorname{Lip}(X)$ and minimal $p$-weak upper gradient $g_{f}$ of $f$ we have

$$
f_{B_{r}}\left|f-f_{B_{r}}\right| d \mu \leq c r f_{B_{\lambda r}} g_{f} d \mu
$$

where

$$
f_{B_{r}}:=f_{B_{r}} f d \mu:=\frac{1}{\mu\left(B_{r}\right)} \int_{B_{r}} f d \mu
$$

is the integral average of $f$ on $B_{r}(x)$.

It is well known that the doubling condition and the Poincaré inequality imply the quasiconvexity of the metric space $X$, see [17] and [13]. Therefore, up to a biLipschitz change of the metric, the space $X$ can be assumed to be geodesic, that is, given $x, y \in X$ there is a curve $\gamma$ with end points $x, y$ and length $d(x, y)$. Moreover, for a geodesic space the weak (1,1)-Poincaré inequality implies the (1,1)-Poincaré inequality, i.e., (2.3) holds with $\lambda=1$. Therefore, as most of the properties of metric spaces we consider are bi-Lipschitz invariant, it is not restrictive to assume that $X$ is a geodesic space and supports a $(1,1)$-Poincaré inequality. 
We remark here that up to Proposition 4.2 assuming only a (1,2)-Poincaré inequality would suffice. However, in Proposition 4.2, and what follows thereafter, a $(1,1)$-Poincaré inequality is needed, for instance, to conclude that the minimal 1 -weak upper gradient is equal $\mu$-a.e. to its pointwise Lipschitz-constant function.

As proved by Cheeger in [7], in our setting the following differentiable structure is given. There exists a countable measurable covering $U_{\alpha}$ of $X$, and Lipschitz coordinate charts $X^{\alpha}=\left(X_{1}^{\alpha}, \ldots, X_{k_{\alpha}}^{\alpha}\right): X \rightarrow \mathbb{R}^{k_{\alpha}}$ such that $\mu\left(U_{\alpha}\right)>0$ for each $\alpha$, $\mu\left(X \backslash \bigcup_{\alpha} U_{\alpha}\right)=0$ and for all $\alpha$ the following holds: the charts $\left(X_{1}^{\alpha}, \ldots, X_{k_{\alpha}}^{\alpha}\right)$ are linearly independent on $U_{\alpha}$ and $1 \leq k_{\alpha} \leq N$, where $N$ is a constant depending on the doubling constant and the constants from the (1,1)-Poincaré inequality satisfying the following condition: For any Lipschitz function $f: X \rightarrow \mathbb{R}$ there is an associated unique (up to a set of zero $\mu$-measure) measurable function $d_{\alpha} f$ : $U_{\alpha} \rightarrow \mathbb{R}^{k_{\alpha}}$ for which the following Taylor-type approximation

$$
f(x)=f\left(x_{0}\right)+d_{\alpha} f\left(x_{0}\right) \cdot\left(X_{\alpha}(x)-X_{\alpha}\left(x_{0}\right)\right)+o\left(d\left(x, x_{0}\right)\right)
$$

holds for $\mu$-a.e. $x_{0} \in U_{\alpha}$.

The previous construction implies, in particular, that for $x \in U_{\alpha}$ there exists a norm $\|\cdot\|_{x}$ on $\mathbb{R}^{k_{\alpha}}$ equivalent to the Euclidean norm $|\cdot|$, such that $g_{f}(x)=$ $\left\|d_{\alpha} f(x)\right\|_{x}$ for almost every $x \in U_{\alpha}$. Moreover, it is possible to show that there exists a constant $c>1$ such that

$$
\frac{1}{c} g_{f}(x) \leq|d f(x)| \leq c g_{f}(x)
$$

for all Lipschitz functions $f$ and $\mu$-a.e. $x \in X$. By $d f(x)$ we mean $d_{\alpha} f(x)$ whenever $x \in U_{\alpha}$. Indeed, one can choose the cover such that $U_{\alpha} \cap U_{\beta}$ is empty whenever $\alpha \neq \beta$.

Formula (2.4) implies in particular linearity of the operator $f \mapsto d f$ and also the Leibniz rule $d(f g)=f d g+g d f$ holds for all Lipschitz functions $f$ and $g$.

For the definition of the Sobolev spaces $N^{1, p}(X, \mu)$ we will follow [23]. Since we assume $X$ to satisfy the $(1,1)$-Poincare inequality, the Sobolev space $N^{1, p}(X, \mu)$, $1 \leq p<\infty$, can also be defined as the closure of the collection of Lipschitz functions on $X$ in the following $N^{1, p}$-norm

$$
\|u\|_{1, p}^{p}=\|u\|_{L^{p}(X)}^{p}+\left\|g_{u}\right\|_{L^{p}(X)}^{p} .
$$

The space $N^{1, p}(X, \mu)$, equipped with the $N^{1, p}$-norm, is a Banach space and a lattice [23].

Let $E \subset X$ be a Borel set. The $p$-capacity of $E$ is defined as usual to be the number

$$
\operatorname{Cap}_{p}(E)=\inf _{u}\left(\int_{X}|u|^{p} d \mu+\int_{X}|d u|^{p} d \mu\right),
$$

where the infimum is taken over all $u \in N^{1, p}(X, \mu)$ for which $u=1$ on $E$. We say that a property holds $p$-quasieverywhere, $p$-q.e. for short, if the set of points for which the property does not hold has $p$-capacity zero. For instance, if $u, v \in$ $N^{1, p}(X, \mu)$ and $u=v \mu$-a.e., then $u=v p$-q.e. and $\|u-v\|_{1, p}=0$. If we, 
moreover, redefine a function $u \in N^{1, p}(X, \mu)$ on a set of zero $p$-capacity, then it remains a representative of the same equivalence class in $N^{1, p}(X, \mu)$.

We shall also use Sobolev spaces defined on a domain $\Omega$ (i.e., a non-empty open pathconnected set) of $X$; the space $N^{1,2}(\Omega, \mu)$ is defined in the same way the space $N^{1,2}(X, \mu)$ is, but considering $\Omega$ as the ambient space. The space of Sobolev functions with zero boundary values is instead defined as

$$
N_{0}^{1, p}(\Omega, \mu)=\left\{u \in N^{1, p}(X, \mu): u=0 p \text {-q.e. on } X \backslash \Omega\right\} .
$$

We have that $N_{0}^{1, p}(\Omega, \mu)=N^{1, p}(X, \mu)$ as Banach spaces if and only if $\operatorname{Cap}_{p}(X)$ $\Omega)=0$.

In what follows, let $p=2$. By [10], the Cheeger differentiable structure extends to all functions in $N^{1,2}(X, \mu)$ and $N^{1,2}(\Omega, \mu)$, and hence we define an inner product on $N^{1,2}(X, \mu)$ by the Dirichlet form

$$
\mathcal{E}(u, v)=\int_{X}\langle d u, d v\rangle d \mu
$$

for all $u, v \in N^{1,2}(X, \mu)$. It can be proved that such a form is strongly regular with the domain, or core, given by $N^{1,2}(X, \mu)$.

We recall that a Dirichlet form is said to be strongly regular if there exists a subset $K$ of the domain of the Dirichlet form, dense in both this domain and in the class of Lipschitz functions on $X$, such that the distance $d_{\mathcal{E}}: X \times X \rightarrow[0, \infty]$ defined, in our case, by

$$
d_{\mathcal{E}}(x, y)=\sup \{\varphi(x)-\varphi(y):|d \varphi(x)| \leq 1\}
$$

is a metric on $X$ that induces the same topology on $X$ as the original metric topology on $X$. In fact, under the doubling property and a Poincaré inequality $d_{\mathcal{E}}$ is bi-Lipschitz equivalent to the original metric $d$ on $X$, and so the Dirichlet form $\mathcal{E}(u, v)$ is strongly regular. The set $K$ is called a core of $\mathcal{E}$. We refer to [25] and [11] for more details.

For each $\alpha>0$ we define the bilinear form

$$
\mathcal{E}_{\alpha}(u, v)=\alpha \int_{X} u v d \mu+\mathcal{E}(u, v) .
$$

We thus have on $N^{1,2}(X, \mu)$ the norm $\|\cdot\|_{\alpha}$ induced by $\mathcal{E}_{\alpha}$ which is equivalent to the $N^{1,2}$-norm. In this way, $N^{1,2}(X, \mu)$ with the norm $\|\cdot\|_{\alpha}$ is a Hilbert space with inner product $\mathcal{E}_{\alpha}$. Note that $\mathcal{E}$ by itself is not an inner product on $N^{1,2}(X, \mu)$; $\mathcal{E}(u, u)=0$ if and only if $u$ is a constant (see [7]). If, for example, $\mu(X)<\infty$, then $\mathcal{E}(u, u)=0$ does not imply that $u=0$.

The fact that the bilinear form $\mathcal{E}_{\alpha}$ yields a Hilbert space can be seen as follows. Since the $N^{1,2}$-norm is comparable to the $\mathcal{E}_{\alpha}$-norm, we have that $N^{1,2}(X, \mu)$ is complete also with respect to the $\mathcal{E}_{\alpha}$-norm. In this way the $\mathcal{E}_{\alpha}$-norm is well defined for any $u \in N^{1,2}(X, \mu)$. By approximation and the linearity of the map $u \mapsto d u$, the Leibniz rule follows for functions $u$ and $v$ in $N^{1,2}(X, \mu)$ (we refer for these properties to the paper [10]). 
Remark 2.1. We point out that the convergence of a sequence $\left(u_{k}\right)_{k}$ to a function $u$ in $N^{1,2}(X, \mu)$ is same as the convergence of the two sequences $\left(u_{k}-u\right)_{k}$ and $\left(g_{u_{k}-u}\right)_{k}$ to 0 in $L^{2}(X, \mu)$.

In general, the convergence of $u_{k}$ to $u$ in $L^{2}(X, \mu)$ together with the convergence of $g_{u_{k}}$ to $g_{u}$ in $L^{2}(X, \mu)$ does not imply that $u_{k}$ converges to $u$ in $N^{1,2}(X, \mu)$. As a counterexample, consider the metric space $X=\mathbb{R}^{2}$ with the distance induced by the norm $\|(x, y)\|_{1}=|x|+|y|$ and with $\mu$ the Lebesgue measure; in this case the upper gradient is determined by the dual norm $\|(x, y)\|_{\infty}=\max \{|x|,|y|\}$. It suffices to verify this for a Lipschitz function $u$. For such function, by Theorem 4.38 in [7], denoting by $B_{r}^{(1)}\left(x_{0}, y_{0}\right)$ the ball in the norm $\|\cdot\|_{1}$ with radius $r$ centered at $\left(x_{0}, y_{0}\right)$, we have that

$$
\begin{aligned}
g_{u}\left(x_{0}, y_{0}\right) & =\lim _{r \rightarrow 0} \sup _{(x, y) \in B_{r}^{(1)}\left(x_{0}, y_{0}\right)} \frac{\left|u(x, y)-u\left(x_{0}, y_{0}\right)\right|}{r} \\
& =\max _{\|v\|_{1}=1}\left\langle\nabla u\left(x_{0}, y_{0}\right), v\right\rangle_{\mathbb{R}^{2}}=\left\|\nabla u\left(x_{0}, y_{0}\right)\right\|_{\infty} .
\end{aligned}
$$

The sequence $u_{k}(x, y)=x+f_{k}(y)$, where $f_{k}(y)=\operatorname{dist}\left(y, \frac{1}{k} \mathbb{Z}\right)$ converges to the function $u(x, y)=x$, but for a.e. point

$$
g_{u_{k}}(x, y)=\left\|\nabla u_{k}(x, y)\right\|_{\infty}=1=\|\nabla u(x, y)\|_{\infty}=g_{u}(x, y)
$$

and

$$
g_{u_{k}-u}(x, y)=\left\|\nabla u_{k}(x, y)-\nabla u(x, y)\right\|_{\infty}=\left\|\nabla f_{k}(y)\right\|_{\infty}=1 .
$$

Nevertheless, it is possible to use Mazur's lemma to prove that for a convex combination the aforementioned property holds true, both for the Cheeger differentiable structure and for the upper gradient. For the Cheeger differentiable structure, however, it is not necessary to take convex combinations. Indeed, in this case the sequence of gradients $d u_{h}$ is bounded in $L^{2}\left(X, \mathbb{R}^{k}, \mu\right)$, and so it is weakly convergent to some $\varphi \in L^{2}\left(X, \mathbb{R}^{k}, \mu\right)$. Mazur's lemma is then needed only to show that $\varphi=d u$. We can consider convex combinations

$$
v_{k}=\sum_{i=1}^{N(k)} \lambda_{i}^{(k)} u_{i}
$$

with strong convergence $v_{k} \rightarrow u$ in $L^{2}(X, \mu)$ and $d v_{k} \rightarrow \varphi$ in $L^{2}\left(X, \mathbb{R}^{k}, \mu\right)$, that is $v_{k} \rightarrow u$ in $N^{1,2}(X, \mu)$, and we may then conclude that $\varphi=d u$. We then obtain

$$
\begin{aligned}
\lim _{k \rightarrow \infty} \int_{X}\left|d u-d u_{k}\right|^{2} d \mu & =\int_{X}|d u|^{2} d \mu+\lim _{k \rightarrow \infty}\left(\int_{X}\left|d u_{k}\right|^{2} d \mu-2 \int_{X}\left\langle d u, d u_{k}\right\rangle d \mu\right) \\
& =2 \int_{X}|d u|^{2} d \mu-2 \int_{X}\langle d u, \varphi\rangle d \mu=0
\end{aligned}
$$

by the weak convergence. 


\section{Metric Laplace operator}

In this section we construct a metric Laplace operator $\Delta_{X}$ on the metric measure space $(X, d, \mu)$. Recall that a Dirichlet form $\mathcal{E}$ is strongly local if whenever $u, v$ are in the domain of $\mathcal{E}$ and $u$ is constant on the support of $v$, then $\mathcal{E}(u, v)=0$. Having a strongly local Dirichlet form at one's disposal it is rather standard argument to construct an operator associated to the form. Most of the statements (without detailed proofs) can be found in the book of Fukushima, Oshima and Takeda [11], but we provide complete proofs for the reader's convenience. Since this operator plays the role of the Laplace operator on $X$, we shall denote it by $\Delta_{X}$. Setting

$$
\begin{aligned}
& \operatorname{Dom}\left(\Delta_{X}\right)=\left\{u \in N^{1,2}(X, \mu): \text { there exists } f \in L^{2}(X, \mu)\right. \\
&\text { such that } \left.\mathcal{E}(u, v)=-\int_{X} f v d \mu \text { for all } v \in N^{1,2}(X, \mu)\right\},
\end{aligned}
$$

the Laplace operator is defined by

$$
\Delta_{X} u=f .
$$

We summarize the main properties of this operator in the following theorem. The main point is to construct the resolvent operator $R_{\alpha}$, i.e., an operator that gives for any $\alpha>0$ the formal solution of the problem

$$
\left(\alpha-\Delta_{X}\right) u=f
$$

and to deduce from this the main properties of $\Delta_{X}$.

Theorem 3.1. For each $\alpha>0$, there is an injective bounded linear operator $R_{\alpha}: L^{2}(X, \mu) \rightarrow N^{1,2}(X, \mu)$ such that for all $v \in N^{1,2}(X, \mu)$

$$
\int_{X} f v d \mu=\mathcal{E}_{\alpha}\left(R_{\alpha} f, v\right)=\mathcal{E}\left(R_{\alpha} f, v\right)+\alpha\left(R_{\alpha} f, v\right)_{2} .
$$

This operator satisfies:

1) for any $f \in L^{2}(X, \mu),\left\|R_{\alpha} f\right\|_{2} \leq \frac{1}{\alpha}\|f\|_{2}$;

2) for any $\alpha, \beta>0, R_{\alpha}\left(L^{2}(X, \mu)\right)=R_{\beta}\left(L^{2}(X, \mu)\right)$, and the resolvent equation holds true

$$
R_{\alpha} f-R_{\beta} f=(\beta-\alpha) R_{\alpha} R_{\beta} f
$$

for all $f \in L^{2}(X, \mu)$;

3) for any $f \in L^{2}(X, \mu)$, we have the following limit in the $L^{2}(X, \mu)$-norm:

$$
\lim _{\alpha \rightarrow \infty} \alpha R_{\alpha} f=f .
$$

Properties 2) and 3) imply that $R_{\alpha}\left(L^{2}(X, \mu)\right)$ is dense in $L^{2}(X, \mu)$. In addition,

$$
\operatorname{Dom}\left(\Delta_{X}\right)=R_{\alpha}\left(L^{2}(X, \mu)\right)
$$

for any $\alpha>0$, and for $u=R_{\alpha} f, \Delta_{X} u:=\alpha u-f$ is independent of $\alpha$. 
Proof. Let us fix $f \in L^{2}(X, \mu)$. Then we can define the linear operator $T_{f}: N^{1,2}(X)$ $\rightarrow \mathbb{R}$ by $T_{f}(v)=(f, v)_{2}:=\int_{X} f v d \mu$. We have that

$$
\left|T_{f}(v)\right| \leq\|f\|_{2}\|v\|_{2} \leq \frac{\|f\|_{2}}{\sqrt{\alpha}} \mathcal{E}_{\alpha}(v, v)^{1 / 2} .
$$

Therefore $T_{f}$ is a bounded linear operator on the Hilbert space $\left(N^{1,2}(X, \mu), \mathcal{E}_{\alpha}\right)$, so by the Riesz representation theorem, there exists an element of $N^{1,2}(X, \mu)$, denoted by $R_{\alpha} f$, such that $T_{f}(v)=\mathcal{E}_{\alpha}\left(R_{\alpha} f, v\right)$. The map $R_{\alpha}: L^{2}(X, \mu) \rightarrow N^{1,2}(X, \mu)$ defined above is linear by the linearity of the defining operator $f \mapsto T_{f}$.

Since

$$
\alpha\left(R_{\alpha} f, v\right)_{2}=\mathcal{E}_{\alpha}\left(R_{\alpha} f, v\right)-\mathcal{E}\left(R_{\alpha} f, v\right)=(f, v)_{2}-\mathcal{E}\left(R_{\alpha} f, v\right),
$$

choosing $v=R_{\alpha} f$ and applying Hölder's inequality, we see that

$$
\begin{aligned}
0 \leq \alpha\left\|R_{\alpha} f\right\|_{2}^{2}=\alpha\left(R_{\alpha} f, R_{\alpha} f\right)_{2} & =\left(f, R_{\alpha} f\right)_{2}-\mathcal{E}\left(R_{\alpha} f, R_{\alpha} f\right) \\
& \leq\left(f, R_{\alpha} f\right)_{2} \leq\|f\|_{2}\left\|R_{\alpha} f\right\|_{2} .
\end{aligned}
$$

Thus we obtain Claim 1 of the theorem, namely,

$$
\alpha\left\|R_{\alpha} f\right\|_{2} \leq\|f\|_{2}
$$

Thus $R_{\alpha}$ as an operator mapping $L^{2}(X, \mu)$ to $L^{2}(X, \mu)$ is bounded with image in $N^{1,2}(X, \mu) \subset L^{2}(X, \mu)$ and its operator norm given by

$$
\left\|R_{\alpha}\right\|:=\left\|R_{\alpha}\right\|_{L^{2} \rightarrow L^{2}} \leq \frac{1}{\alpha}
$$

We now prove the resolvent equation (3.2). Let us take $f \in L^{2}(X, \mu)$ and $v \in N^{1,2}(X, \mu)$. Then

$$
\begin{aligned}
\mathcal{E}_{\alpha}\left(R_{\alpha} f-R_{\beta} f\right. & \left.+(\alpha-\beta) R_{\alpha} R_{\beta} f, v\right) \\
& =\mathcal{E}_{\alpha}\left(R_{\alpha} f, v\right)-\mathcal{E}_{\alpha}\left(R_{\beta} f, v\right)+(\alpha-\beta) \mathcal{E}_{\alpha}\left(R_{\alpha} R_{\beta} f, v\right) \\
& =(f, v)_{2}-\mathcal{E}\left(R_{\beta} f, v\right)-\alpha\left(R_{\beta} f, v\right)_{2}+(\alpha-\beta)\left(R_{\beta} f, v\right)_{2} \\
& =(f, v)_{2}-\mathcal{E}_{\beta}\left(R_{\beta} f, v\right)=0 .
\end{aligned}
$$

This means that for $f \in N^{1,2}(X, \mu)$, and then by density also for $f \in L^{2}(X, \mu)$, we have the identity

$$
R_{\alpha} f-R_{\beta} f+(\alpha-\beta) R_{\alpha} R_{\beta} f=0 .
$$

Moreover, if we consider $f \in N^{1,2}(X, \mu)$, we have (denoting $\mathcal{E}_{\alpha}(f, f)^{1 / 2}=:\|f\|_{\alpha}$ )

$$
\begin{aligned}
\alpha\left\|\alpha R_{\alpha} f-f\right\|_{2}^{2} & \leq \mathcal{E}_{\alpha}\left(\alpha R_{\alpha} f-f, \alpha R_{\alpha} f-f\right) \\
& =\alpha^{2} \mathcal{E}_{\alpha}\left(R_{\alpha} f, R_{\alpha} f\right)+\|f\|_{\alpha}^{2}-2 \alpha \mathcal{E}_{\alpha}\left(R_{\alpha} f, f\right) \\
& =\alpha^{2}\left(f, R_{\alpha} f\right)_{2}+\mathcal{E}(f, f)-\alpha\|f\|_{2}^{2} .
\end{aligned}
$$


By Hölder's inequality and by using (3.4), we also get

$$
\alpha\left(f, R_{\alpha} f\right)_{2}-\|f\|_{2}^{2} \leq \alpha\left\|R_{\alpha} f\right\|_{2}\|f\|_{2}-\|f\|_{2}^{2} \leq 0 .
$$

Therefore,

$$
\lim _{\alpha \rightarrow \infty}\left\|\alpha R_{\alpha} f-f\right\|_{2} \leq \lim _{\alpha \rightarrow \infty} \sqrt{\frac{\mathcal{E}(f, f)}{\alpha}}=0 .
$$

To extend this limit to be valid for any $f \in L^{2}(X, \mu)$, we use the boundedness of $R_{\alpha}$, by fixing $f_{\varepsilon} \in N^{1,2}(X, \mu)$ such that $\left\|f-f_{\varepsilon}\right\|_{2} \leq \varepsilon$. In this way we get that

$$
\begin{aligned}
\left\|\alpha R_{\alpha} f-f\right\|_{2} & \leq\left\|\alpha R_{\alpha} f_{\varepsilon}-f_{\varepsilon}\right\|_{2}+\alpha\left\|R_{\alpha}\left(f-f_{\varepsilon}\right)\right\|_{2}+\left\|f-f_{\varepsilon}\right\|_{2} \\
& \leq\left\|\alpha R_{\alpha} f_{\varepsilon}-f_{\varepsilon}\right\|_{2}+2 \varepsilon,
\end{aligned}
$$

and hence

$$
\limsup _{\alpha \rightarrow 0}\left\|\alpha R_{\alpha} f-f\right\|_{2} \leq 2 \varepsilon .
$$

From this Claim 3 of the theorem follows since $\varepsilon$ was arbitrary.

We have now proved that $R_{\alpha}$ is a strongly continuous resolvent (see [11]) for any $\alpha>0$. Let us next prove injectivity of $R_{\alpha}$. Suppose $f \in L^{2}(X, \mu)$ is such that $R_{\beta} f=0$ for some $\beta>0$. Then by the resolvent equation (3.2),

$$
0=R_{\alpha} f-R_{\beta} f+(\alpha-\beta) R_{\alpha} R_{\beta} f=R_{\alpha} f,
$$

that is, $R_{\alpha} f=0$ for every $\alpha>0$. Now by equation (3.3), we see that $f=0$, that is, $R_{\alpha}$ is injective. We can therefore define the inverse map $R_{\alpha}^{-1}: R_{\alpha}\left(L^{2}(X, \mu)\right) \rightarrow$ $L^{2}(X, \mu)$. We claim that

$$
\operatorname{Dom}\left(\Delta_{X}\right)=R_{\alpha}\left(L^{2}(X, \mu)\right), \quad \Delta_{X} u=\alpha u-R_{\alpha}^{-1} u .
$$

For this definition to be consistent, we first show that the set $R_{\alpha}\left(L^{2}(X, \mu)\right)$ and the operator $A_{\alpha} u:=\alpha u-R_{\alpha}^{-1} u$ do not depend on $\alpha$. By the resolvent equation (3.2),

$$
R_{\beta} f=R_{\alpha}\left(f+(\alpha-\beta) R_{\beta} f\right) .
$$

Therefore, for every $f \in L^{2}(X, \mu), R_{\beta} f \in R_{\alpha}\left(L^{2}(X, \mu)\right)$, and hence

$$
R_{\beta}\left(L^{2}(X, \mu)\right) \subset R_{\alpha}\left(L^{2}(X, \mu)\right) .
$$

By the symmetry of the argument, we have the required result $R_{\alpha}\left(L^{2}(X, \mu)\right)=$ $R_{\beta}\left(L^{2}(X, \mu)\right)$. Let us write $D=R_{\beta}\left(L^{2}(X, \mu)\right)$.

If $u \in D$ and $\alpha, \beta>0$, then $A_{\alpha} u-A_{\beta} u=(\alpha-\beta) u-R_{\alpha}^{-1} u+R_{\beta}^{-1} u$. Therefore,

$$
R_{\alpha}\left(A_{\alpha} u-A_{\beta} u\right)=\alpha R_{\alpha} u-\beta R_{\alpha} u-u+R_{\alpha} R_{\beta}^{-1} u .
$$

On the other hand, since $D=R_{\beta}\left(L^{2}(X, \mu)\right)$, there exists $f \in L^{2}(X, \mu)$ such that $R_{\beta} f=u$. Hence we have, by the resolvent equation (3.2), that

$$
\begin{aligned}
R_{\alpha}\left(A_{\alpha} u-A_{\beta} u\right) & =\alpha R_{\alpha} R_{\beta} f-\beta R_{\alpha} R_{\beta} f-R_{\beta} f+R_{\alpha} f \\
& =R_{\alpha} f-R_{\beta} f+(\alpha-\beta) R_{\alpha} R_{\beta} f=0 .
\end{aligned}
$$

By injectivity of $R_{\alpha}$, we see that $A_{\alpha} u-A_{\beta} u=0$, i.e., $A_{\alpha} u=A_{\beta} u$. 
Let us now show that $R_{\alpha}\left(L^{2}(X, \mu)\right) \subset \operatorname{Dom}\left(\Delta_{X}\right)$. Let $u \in R_{\alpha}\left(L^{2}(X, \mu)\right)$. Then there exists $f \in L^{2}(X, \mu)$ such that $u=R_{\alpha} f$. The identity $\mathcal{E}_{\alpha}\left(R_{\alpha} f, v\right)=(f, v)_{2}$ for any $v \in N^{1,2}(X, \mu)$ can be written out as follows:

$$
\begin{aligned}
\int_{X}\langle d u, d v\rangle d \mu & =\mathcal{E}(u, v)=\mathcal{E}_{\alpha}(u, v)-\alpha(u, v)_{2}=\mathcal{E}_{\alpha}\left(R_{\alpha} f, v\right)-\alpha(u, v)_{2} \\
& =(f, v)_{2}-\alpha(u, v)_{2}=-\int_{X}(\alpha u-f) v d \mu
\end{aligned}
$$

for all $v \in N^{1,2}(X, \mu)$. This simply means that $u \in \operatorname{Dom}\left(\Delta_{X}\right)$ and that $\Delta_{X} u=$ $\alpha u-f=\alpha u-R_{\alpha}^{-1} u=A_{\alpha} u$.

For the reverse inclusion, $\operatorname{Dom}\left(\Delta_{X}\right) \subset R_{\alpha}\left(L^{2}(X, \mu)\right)$, let us consider $u \in$ $\operatorname{Dom}\left(\Delta_{X}\right)$. Thus there exists $f \in L^{2}(X, \mu)$ such that for all $v \in N^{1,2}(X, \mu)$ we have

$$
\int_{X}\langle d u, d v\rangle d \mu=-\int_{X} f v d \mu
$$

Then consider $w:=R_{\alpha}(\alpha u-f)$; we obtain that

$\mathcal{E}_{\alpha}(w, v)=(\alpha u-f, v)_{2}=\alpha \int_{X} u v d \mu-\int_{X} f v d \mu=\alpha \int_{X} u v d \mu+\mathcal{E}(u, v)=\mathcal{E}_{\alpha}(u, v)$,

that is $w=u$, which means that $u \in R_{\alpha}\left(L^{2}(X, \mu)\right)$. The identity $f=\Delta_{X} u=$ $\alpha u-R_{\alpha}^{-1} u$ follows easily.

In addition to the density of $\operatorname{Dom}\left(\Delta_{X}\right)$ in $L^{2}(X, \mu)$, we also have that $\operatorname{Dom}\left(\Delta_{X}\right)$ is dense in $N^{1,2}(X, \mu)$. In fact, by (3.4) and (3.5), for any $f \in N^{1,2}(X, \mu)$, we have that

$$
\begin{aligned}
\left\|\alpha R_{\alpha} f-f\right\|_{\alpha}^{2} & =\mathcal{E}_{\alpha}\left(\alpha R_{\alpha} f-f, \alpha R_{\alpha} f-f\right) \\
& =\alpha^{2} \mathcal{E}_{\alpha}\left(R_{\alpha} f, R_{\alpha} f\right)-2 \alpha \mathcal{E}_{\alpha}\left(R_{\alpha} f, f\right)+\mathcal{E}_{\alpha}(f, f) \\
& =\alpha^{2}\left(f, R_{\alpha} f\right)_{2}-\alpha(f, f)_{2}+\mathcal{E}(f, f) \leq \mathcal{E}(f, f),
\end{aligned}
$$

that is the sequence $\left(\alpha R_{\alpha} f-f\right)_{\alpha}$ is bounded in $N^{1,2}(X, \mu)$. Therefore, for any sequence of positive real numbers $\left(\alpha_{n}\right)_{n}$ so that $\lim _{n} \alpha_{n}=\infty$, the corresponding sequence of functions $\alpha_{n} R_{\alpha_{n}} f-f$ is a bounded sequence, and hence by Mazur's lemma we have a sequence of convex combinations converging in $N^{1,2}(X, \mu)$;

$$
\left(\sum_{i=n}^{N(n)} \lambda_{i, n} \alpha_{i} R_{\alpha_{i}} f\right)-f \rightarrow w \in N^{1,2}(X, \mu) .
$$

On the other hand, $\lim _{\alpha \rightarrow \infty} \alpha R_{\alpha} f=f$ in $L^{2}(X, \mu)$. Thus $w=0 \mu$-a.e. in $X$, and hence by the fact that $w \in N^{1,2}(X, \mu)$ we know that $w=0 p$-q.e. in $X$. Therefore, it must be that $w=0$. Observe that the sequence of convex combinations $\sum_{i=n}^{N(n)} \lambda_{i, n} \alpha_{i} R_{\alpha_{i}} f$ lies in $\operatorname{Dom}\left(\Delta_{X}\right)$ and converges to $f \in N^{1,2}(X, \mu)$, so the proof is completed.

We can now give the definition of a Cheeger harmonic function in the obvious way. 
Definition 3.2. A function $u \in N^{1,2}(\Omega, \mu)$ is said to be Cheeger harmonic (referred to in this paper as harmonic) if

$$
\int_{\Omega}\langle d u, d v\rangle d \mu=0
$$

for all $v \in N_{0}^{1,2}(\Omega, \mu)$, i.e., $u$ is harmonic if and only if $u \in \operatorname{Dom}\left(\Delta_{\Omega}\right)$ and $\Delta_{\Omega} u=0$. Here $\Delta_{\Omega}$ is the operator defined in Remark 3.4 below.

Remark 3.3. The notion of Cheeger harmonicity refers to the fact that we are using the Cheeger differentiable structure. This notion has been previously considered in the paper [19], where Lipschitz regularity of Cheeger harmonic functions has been investigated. We also underline that Cheeger harmonicity can be equivalently be given in terms of a minimizer of the Dirichlet energy: $u$ is Cheeger harmonic if and only if for any ball $B_{r}$

$$
\int_{B_{r}}|d u|^{2} d \mu \leq \int_{B_{r}}|d v|^{2} d \mu,
$$

for all $v$ such that $v-u \in N_{0}^{1,2}\left(B_{r}, \mu\right)$.

Remark 3.4. Let $\Omega \subset X$ be a bounded domain satisfying a (1,2)-Poincaré inequality with $\operatorname{Cap}_{2}(X \backslash \Omega)>0$. The previous construction of $\Delta_{X}$ can also be used to construct a Laplace operator on the subdomain $\Omega$. There are essentially two different Laplace operators; the first is just the restriction of $\Delta_{X}$ to $\Omega$ and is defined by

$$
\begin{array}{r}
\operatorname{Dom}\left(\Delta_{\Omega}\right)=\left\{u \in N^{1,2}(\Omega, \mu): \text { there exists } f \in L^{2}(\Omega, \mu)\right. \text { such that } \\
\left.\int_{\Omega}\langle d u, d v\rangle d \mu=-\int_{\Omega} f v d \mu \text { for all } v \in N_{0}^{1,2}(\Omega, \mu)\right\},
\end{array}
$$

and the operator is given by

$$
\Delta_{\Omega} u=f .
$$

The second alternative, adapted to the inhomogeneous Dirichlet problem, is the operator defined by

$$
\begin{aligned}
& \operatorname{Dom}\left(\Delta_{\Omega}^{D}\right)=\left\{u \in N_{0}^{1,2}(\Omega, \mu) \text { : there exists } f \in L^{2}(\Omega, \mu)\right. \text { such that } \\
& \left.\int_{\Omega}\langle d u, d v\rangle d \mu=-\int_{\Omega} f v d \mu \text { for all } v \in N_{0}^{1,2}(\Omega, \mu)\right\},
\end{aligned}
$$

and

$$
\Delta_{\Omega}^{D} u=f
$$

To define the latter operator, the previous procedure has to be modified by considering the Hilbert space $N_{0}^{1,2}(\Omega, \mu)$ with the inner product $\mathcal{E}_{\alpha}$ for all $\alpha>0$, to obtain the resolvent operator $R_{\alpha}^{0}: N_{0}^{1,2}(\Omega, \mu) \rightarrow N_{0}^{1,2}(\Omega, \mu)$ with

$$
\mathcal{E}_{\alpha}\left(R_{\alpha}^{0} f, v\right)=(f, v)_{2}
$$


whenever $v \in N_{0}^{1,2}(\Omega, \mu)$. Since the vector subspace $\operatorname{Lip}_{0}(\Omega)$ of $N_{0}^{1,2}(\Omega, \mu)$ is also a dense subspace of $L^{2}(\Omega, \mu)$, we may extend $R_{\alpha}^{0}$ to be an injective map from $L^{2}(\Omega)$ to $N_{0}^{1,2}(\Omega)$. These properties, like the one proved for $R_{\alpha}$ in Theorem 3.1, are the essential properties for the definition of the operator $\Delta_{\Omega}^{D}$.

It is easy to verify that the operator $\Delta_{\Omega}$ is the restriction of $\Delta_{X}$ to $\Omega$ in the following sense: If $u \in \operatorname{Dom}\left(\Delta_{X}\right)$, then

$$
\Delta_{\Omega}\left(u_{\mid \Omega}\right)=\left(\Delta_{X} u\right)_{\mid \Omega} .
$$

On the other hand, the operator $\Delta_{\Omega}^{D}$ is the restriction of $\Delta_{\Omega}$ to the space $N_{0}^{1,2}(\Omega, \mu)$, that is

$$
\operatorname{Dom}\left(\Delta_{\Omega}^{D}\right)=\operatorname{Dom}\left(\Delta_{\Omega}\right) \cap N_{0}^{1,2}(\Omega, \mu)
$$

with $\Delta_{\Omega}^{D} u=\Delta_{\Omega} u$ for $u \in \operatorname{Dom}\left(\Delta_{\Omega}\right) \cap N_{0}^{1,2}(\Omega, \mu)$.

\subsection{Measure-valued Laplace operator}

Let $\Omega$ be a domain in $X$. We give the following definition of the measure-valued Laplace operator $\mathscr{D}_{\Omega}$ on $\Omega$. By $\mathscr{M}_{b}(\Omega)$ we denote the space of all bounded signed Borel measures on $\Omega$, i.e., $\nu \in \mathscr{M}_{b}(\Omega)$ is a real-valued signed Borel measure on $\Omega$ with bounded total variation

$$
|\nu|(\Omega)=\sup \left\{\int_{\Omega} \varphi d \nu: \varphi \in \operatorname{Lip}_{c}(\Omega),\|\varphi\|_{\infty} \leq 1\right\}<\infty .
$$

We remark that to compute the total variation of a measure we test in the space $\operatorname{Lip}_{c}(\Omega)$ of Lipschitz functions on $\Omega$ with compact support instead of the space $C_{c}(\Omega)$ of continuous functions with compact support; we may do this since $\operatorname{Lip}_{c}(\Omega)$ is clearly dense in $C_{c}(\Omega)$.

We define

$$
\operatorname{Dom}\left(\mathscr{D}_{\Omega}\right)=\left\{u \in N^{1,2}(\Omega, \mu): \text { there exists } \nu \in \mathscr{M}_{b}(X)\right. \text { such that }
$$

$$
\left.\mathcal{E}(u, v)=-\int_{\Omega} v d \nu \text { for all } v \in \operatorname{Lip}_{c}(\Omega)\right\},
$$

and then we set

$$
\mathscr{D}_{\Omega} u=\nu \text {. }
$$

Example 3.5. As an example, we can consider the Euclidean space $\left(\mathbb{R}^{n},\|\cdot\|\right)$ and modify its metric structure in two ways, which essentially lead to the same metric measure structure. We fix $\Omega \subset \mathbb{R}^{n}$ an open set with regular boundary and we can modify either the measure by considering $d \mu=\left(1+\chi_{\Omega}\right) d \mathcal{L}^{n}$, or the differential structure $d u=\left(1+\alpha \chi_{\Omega}\right) \nabla u$, where $\alpha=\sqrt{2}-1$.

In both cases we have, for $u, v \in C_{c}^{2}\left(\mathbb{R}^{n}\right)$,

$$
\begin{aligned}
\int_{\mathbb{R}^{n}}\langle d u, d v\rangle d \mu & =\int_{\mathbb{R}^{n}} \nabla u \cdot \nabla v d x+\int_{\Omega} \nabla u \cdot \nabla v d x \\
& =-\int_{\mathbb{R}^{n}} v \Delta u d x-\int_{\Omega} v \Delta u d x+\int_{\partial \Omega} v \nabla u \cdot \nu_{\Omega} d \mathcal{H}^{n-1} .
\end{aligned}
$$

Then $u \in \operatorname{Dom}\left(\Delta_{\mathbb{R}^{n}}\right)$ if and only if $\nabla u \cdot \nu_{\Omega}=0$ on $\partial \Omega$ and $\Delta u \in L^{2}\left(\mathbb{R}^{n}\right)$. 
In addition, in the case $\mu=\left(1+\chi_{\Omega}\right) \mathcal{L}^{n}$ with the standard differential structure we also have

$$
\Delta_{\mathbb{R}^{n}} u=\Delta u \text {. }
$$

In the second case, where $\mu=\mathcal{L}^{n}$ and $d u=\left(1+\alpha \chi_{\Omega}\right) \nabla u$, we obtain

$$
\Delta_{\mathbb{R}^{n}} u=\left(1+\chi_{\Omega}\right) \Delta u \text {. }
$$

In a similar fashion, $\operatorname{Dom}\left(\mathscr{D}_{\mathbb{R}^{n}}\right)$ is given by those functions $u$ for which $\Delta u \in$ $L^{1}\left(\mathbb{R}^{n}\right)$ and the trace of $\nabla u \cdot \nu_{\Omega} \in L^{1}\left(\partial \Omega, \mathcal{H}^{n-1}\right)$, and

$$
\mathscr{D}_{\mathbb{R}^{n}} u=\Delta u \mu-\nabla u \cdot \nu_{\Omega} \mathcal{H}^{n-1}\llcorner\partial \Omega .
$$

It can be verified that $\operatorname{Dom}\left(\mathscr{D}_{\Omega}\right)$ is a vector space and that $\mathscr{D}_{\Omega}$ is linear. We wish to expand the class of test functions in the definition of the domain $\operatorname{Dom}(\mathscr{D} \Omega)$ from $\operatorname{Lip}_{c}(\Omega)$ to allow for test-functions $v$ in $N_{0}^{1,2}(\Omega, \mu)$, see Proposition 3.8. For that, we need the following lemma.

Lemma 3.6. If $E \subset \Omega$ is a Borel set such that $\operatorname{Cap}_{2}(E)=0$, then for every $u \in \operatorname{Dom}\left(\mathscr{D}_{\Omega}\right),\left|\mathscr{D}_{\Omega} u\right|(E)=0$.

Proof. By the Jordan decomposition theorem, the measure $\mathscr{D} \Omega u$ can be decomposed into its positive and negative parts, $\mathscr{D}_{\Omega}^{+} u$ and $\mathscr{D}_{\Omega}^{-} u$; this means that we can decompose $\Omega$ into two disjoint Borel sets $\Omega=\Omega^{+} \cup \Omega^{-}$in such a way that $\mathscr{D} \Omega u(B) \geq 0$ for every $B \subset \Omega^{+}$and $\mathscr{D} \Omega u(B) \leq 0$ for every $B \subset \Omega^{-}$. Hence we may, without loss of generality, consider $E \subset \Omega^{+}$; in fact we can decompose $E=E^{+} \cup E^{-}$and use the monotonicity of capacity. Further, we may also assume that $E$ is a compact set, since as Radon measures both $\mathscr{D}_{\Omega}^{+} u$ and $\mathscr{D} \bar{\Omega}^{-} u$ are inner measures and $E$ is a Borel set.

Since $\operatorname{Cap}_{2}(E)=0$, we have also that the relative capacity $\operatorname{Cap}_{2}(E, \Omega)$ is zero. This can be seen by multiplying those Lipschitz test-functions which were used for computing $\operatorname{Cap}_{2}(E)$ by another Lipschitz function $\eta$ which is 1 on a neighborhood of the compact set $E$ and has compact support in $\Omega$. We can then find a sequence of Lipschitz functions $\left(\varphi_{i}\right)_{i}$ so that $0 \leq \varphi_{i} \leq 1$ on $X, \varphi_{i}=1$ on $E$, and $\left\|\varphi_{i}\right\|_{N^{1,2}(X)} \leq$ $2^{-i}$, and $\varphi_{i}$ are compactly supported in $\Omega$. We may assume that the sequence $\left(\varphi_{i}\right)_{i}$ converges pointwise to zero outside of the compact set $E$ (we can do so by choosing $\varphi_{i}$ to have support in the open set $\left.\bigcup_{x \in E} B(x, 1 / i)\right)$. We have

$$
\begin{aligned}
\left|\int_{X} \varphi_{i} d \mathscr{D} \Omega u\right| & =\left|\int_{X}\left\langle d u, d \varphi_{i}\right\rangle d \mu\right| \leq\left(\int_{X}|d u|^{2} d \mu\right)^{1 / 2}\left(\int_{X}\left|d \varphi_{i}\right|^{2} d \mu\right)^{1 / 2} \\
& \leq\left(\int_{X}|d u|^{2} d \mu\right)^{1 / 2}\left\|\varphi_{i}\right\|_{N^{1,2}(X, \mu)},
\end{aligned}
$$

which tends to 0 as $i \rightarrow \infty$.

On the other hand, since $\varphi_{i}$ are all bounded by 1 and $\left|\mathscr{D}_{\Omega}\right|(X)<\infty$, by the Lebesgue dominated convergence theorem we have

$$
\lim _{i \rightarrow \infty} \int_{X} \varphi_{i} d \mathscr{D}_{\Omega} u=\mathscr{D}_{\Omega} u(E)=\mathscr{D}_{\Omega}^{+} u(E) .
$$

A similar argument shows that $\mathscr{D}_{\Omega}^{-} u(E)=0$, and hence the proof follows. 
Remark 3.7. The requirement that $E$ is a Borel set in the above lemma is not a serious restriction, because if $E \subset \Omega$ is a set with $\operatorname{Cap}_{2}(E)=0$, then there is a Borel set $E_{0}$ with $E \subset E_{0} \subset \Omega$ such that $\operatorname{Cap}_{2}\left(E_{0}\right)=0$.

The following proposition tells us that we do not have to restrict ourselves to having test-functions $v$ only in $\operatorname{Lip}_{c}(X)$ in (3.6).

Proposition 3.8. Let $u \in \operatorname{Dom}(\mathscr{D} \Omega)$. Then for every $v \in N_{0}^{1,2}(\Omega, \mu) \cap L^{\infty}(\Omega, \mu)$ the following holds:

$$
\mathcal{E}(u, v)=-\int_{\Omega} v d \mathscr{D} \Omega u
$$

Proof. We first assume that $v$ has compact support in $\Omega$. Note that by the $(1,2)$-Poincaré inequality we can approximate compactly supported functions in $N^{1,2}(\Omega, \mu)$ by Lipschitz functions. So we can find a sequence of compactly supported Lipschitz functions $\left(\varphi_{i}\right)_{i}$ on $\Omega$ that converge to $v$ in the $N^{1,2}(\Omega, \mu)$-norm. By passing to a subsequence if necessary, we may also assume that $\varphi_{i} \rightarrow v$ pointwise outside a set of zero 2-capacity; we refer to [23]. Since $v$ is bounded, we can also assume that the approximating compactly supported Lipschitz functions $\varphi_{i}$ are also uniformly bounded by $M:=\|v\|_{\infty}$. Applying $\varphi_{i}$ as in (3.6), we see that

$$
\int_{\Omega} \varphi_{i} d \mathscr{D} \Omega u=-\int_{\Omega}\left\langle d \varphi_{i}, d u\right\rangle d \mu \rightarrow-\int_{\Omega}\langle d v, d u\rangle d \mu .
$$

By Lemma 3.6, we know that $\varphi_{i} \rightarrow v$ almost everywhere with respect to the total variation measure $\left|\mathscr{D}_{\Omega} u\right|$. By the Lebesgue dominated convergence theorem applied to the uniformly bounded functions $\varphi_{i}$ with respect to the positive and negative parts $\mathscr{D} \Omega u^{+}, \mathscr{D} \Omega u^{-}$of the signed Borel measure $\mathscr{D} \Omega u$, we may conclude that

$$
\int_{\Omega} \varphi_{i} d \mathscr{D}_{\Omega} u \rightarrow \int_{\Omega} v d \mathscr{D} \Omega u
$$

Hence equation (3.6) holds for all compactly supported functions $v \in N^{1,2}(\Omega, \mu) \cap$ $L^{\infty}(\Omega, \mu)$.

To pass to any $v \in N_{0}^{1,2}(\Omega, \mu) \cap L^{\infty}(\Omega, \mu)$, we note that functions in $N_{0}^{1,2}(\Omega, \mu)$ with compact support in $\Omega$ form a dense subclass of $N_{0}^{1,2}(\Omega, \mu)$ (see [24]). Hence, if $v$ is in $N_{0}^{1,2}(\Omega, \mu) \cap L^{\infty}(\Omega, \mu)$, we can find a sequence of compactly supported functions $v_{i}$ from $N_{0}^{1,2}(\Omega, \mu) \cap L^{\infty}(\Omega, \mu)$ such that $v_{i} \rightarrow v$ in $N_{0}^{1,2}(\Omega, \mu)$. As before, we can also ensure that $v_{i} \rightarrow v 2$-capacity almost everywhere in $\Omega$. Hence

$$
\int_{\Omega}\langle d u, d v\rangle d \mu=\lim _{i \rightarrow \infty} \int_{\Omega}\left\langle d u, d v_{i}\right\rangle d \mu=-\lim _{i \rightarrow \infty} \int_{\Omega} v_{i} d \mathscr{D}_{\Omega} u,
$$

and then if $v$ is bounded in $\Omega$ we have

$$
\lim _{i \rightarrow \infty} \int_{\Omega} v_{i} d \mathscr{D} \Omega u=\int_{\Omega} v d \mathscr{D} \Omega u,
$$

giving the desired result for all bounded functions in $N_{0}^{1,2}(\Omega, \mu)$. 
We shall also need the following lemma, which is based on the Lebesgue decomposition of the measure $\mathscr{D}_{\Omega}$ given by

$$
d \mathscr{D}_{\Omega} u=f_{u} d \mu+d \mathscr{D}_{\Omega}^{s} u
$$

where $f_{u}=d \mathscr{D}_{\Omega} u / d \mu$ is the absolutely continuous part and $\mathscr{D}_{\Omega}^{s}$ the singular part of $\mathscr{D} \Omega$.

Lemma 3.9. Let $u \in \operatorname{Dom}\left(\mathscr{D}_{\Omega}\right)$. If the singular part $\mathscr{D}_{\Omega}^{s}$ of $\mathscr{D}_{\Omega} u$ is zero and if the Radon-Nikodym derivative $f_{u} \in L^{2}(\Omega, \mu)$, then $u \in \operatorname{Dom}\left(\Delta_{\Omega}\right)$ with $\Delta_{\Omega} u=f_{u}$.

Proof. From the discussion in Section 3, if $\mathscr{D}_{\Omega}^{s} u=0$ and the absolutely continuous part is represented by $f_{u} \in L^{2}(\Omega, \mu)$, then

$$
\mathcal{E}(u, v)=-\int_{\Omega} f_{u} v d \mu
$$

for all $v \in N_{0}^{1,2}(\Omega, \mu) \cap L^{\infty}(\Omega, \mu)$. When $f_{u} \in L^{2}(\Omega, \mu)$, we can use a truncation argument and the Lebesgue dominated convergence theorem to show that (3.7) holds for any $v \in N_{0}^{1,2}(\Omega, \mu)$. So we conclude that $u \in \operatorname{Dom}\left(\Delta_{\Omega}\right)$ and $\Delta_{\Omega} u=f_{u}$.

Remark 3.10. It can be seen that $\operatorname{Dom}\left(\Delta_{\Omega}\right) \subset \operatorname{Dom}\left(\mathscr{D}_{\Omega}\right)$; moreover, if $u, v \in$ $\operatorname{Dom}\left(\mathscr{D}_{\Omega}\right)$ and $a \in \mathbb{R}$, the following hold true:

1) $\operatorname{spt}\left(\mathscr{D}_{\Omega} u\right) \subset \operatorname{spt}(u)$; also, if $u$ is constant on an open set $U$, then $\operatorname{spt}\left(\mathscr{D}_{\Omega} u\right) \subset$ $\Omega \backslash U$;

2) $u+v, a u \in \operatorname{Dom}\left(\mathscr{D}_{\Omega}\right)$ with $\mathscr{D}_{\Omega}(u+v)=\mathscr{D}_{\Omega} u+\mathscr{D}_{\Omega} v$ and $\mathscr{D}_{\Omega}(a u)=a \mathscr{D}_{\Omega} u$;

3 ) if in addition $u$ and $v$ are bounded, then $u v \in \operatorname{Dom}\left(\mathscr{D}_{\Omega}\right)$ with

$$
d \mathscr{D}_{\Omega}(u v)=v d \mathscr{D}_{\Omega} u+u d \mathscr{D} \Omega v+2\langle d u, d v\rangle d \mu .
$$

Note here that since $u, v$ are in $N^{1,2}(\Omega)$, it follows that they are well-defined up to sets of $\mathrm{Cap}_{2}$-zero; such null sets are not charged by $\mathscr{D} \Omega u, \mathscr{D} \Omega v$, see Lemma 3.6.

\subsection{Inhomogeneous Dirichlet problem}

In this section we consider the inhomogeneous Dirichlet problem on bounded open domains $\Omega$ such that $\mu(X \backslash \Omega)>0$; we assume that a metric space $X$ satisfies a $(1,2)$-Poincaré inequality. More precisely, given two functions $f \in L^{2}(X, \mu)$ and $v \in N^{1,2}(X, \mu)$, we wish to find $u \in \operatorname{Dom}\left(\Delta_{\Omega}\right)$ such that

$$
\left\{\begin{array}{l}
\Delta_{\Omega} u=f \text { on } \Omega, \\
u-v \in N_{0}^{1,2}(\Omega, \mu) .
\end{array}\right.
$$

By definition of $\Delta_{\Omega}$, we interpret (3.8) in the weak sense, i.e., $u$ is a solution of (3.8) if $u-v \in N_{0}^{1,2}(\Omega, \mu)$ and for all $\varphi \in N_{0}^{1,2}(\Omega, \mu)$,

$$
\mathcal{E}(u, \varphi)=-\int_{\Omega} f \varphi d \mu .
$$


As in the classical linear theory, a solution $u$ to (3.8) can be written as the sum of two functions, $u_{0}$ and $u_{1}$, where $u_{0}$ is harmonic in $\Omega$ such that $u_{0}-v \in N_{0}^{1,2}(\Omega, \mu)$ and $u_{1} \in N_{0}^{1,2}(\Omega, \mu)$ is a particular solution to the problem $\Delta_{\Omega}^{D} u_{1}=f$ with $u_{1} \in N_{0}^{1,2}(\Omega, \mu)$.

The function $u_{0}$ is constructed in [19] as the minimum of the energy functional

$$
\min _{u-v \in N_{0}^{1,2}(\Omega, \mu)} \int_{\Omega}|d u|^{2} d \mu .
$$

For the second part, we use the functional $F: N_{0}^{1,2}(\Omega, \mu) \rightarrow \mathbb{R}$ given by

$$
F(u)=\frac{1}{2} \int_{\Omega}|d u|^{2} d \mu+\int_{\Omega} u f d \mu,
$$

which is the sum of a linear functional and a strictly convex energy. Hence $F$ itself is strictly convex. Then, if $F$ has a minimum, it is unique and the minimum is the desired solution $u_{1}$. To prove the existence, it is enough to use the Sobolev inequality, i.e., if $u \in N_{0}^{1,2}(\Omega, \mu)$ there exists a constant $c_{s}>0$ such that

$$
\|u\|_{2} \leq c_{s}\|d u\|_{2}
$$

Given that $\Omega$ is bounded and $\mu(X \backslash \Omega)>0$, the above Sobolev inequality holds; we refer to [13] and [18] for the details. Then, for any $u \in N_{0}^{1,2}(\Omega, \mu)$ we have that, using the inequality $a b \leq \varepsilon a^{2} / 2+b^{2} / 2 \varepsilon$ with $a, b, \varepsilon>0$,

$$
\begin{aligned}
F(u) & =\frac{1}{2}\|d u\|_{2}^{2}+\int_{\Omega} u f d \mu \geq \frac{1}{2}\|d u\|_{2}^{2}-\|f\|_{2}\|u\|_{2} \geq \frac{1}{2}\|d u\|_{2}^{2}-c_{s}\|f\|_{2}\|d u\|_{2} \\
& \geq\left(\frac{1}{2}-\frac{\varepsilon c_{s}}{2}\right)\|d u\|_{2}^{2}-\frac{c_{s}}{2 \varepsilon}\|f\|_{2}^{2} .
\end{aligned}
$$

If we fix $\varepsilon<1 / c_{s}$, the preceding inequality gives us that $F$ is bounded from below by $-2^{-1} \varepsilon^{-1} c_{s}\|f\|_{2}^{2}$. Therefore,

$$
m=\inf _{u \in N_{0}^{1,2}(\Omega, \mu)} F(u)
$$

is finite, and in particular, the infimum is a minimum as seen by taking a minimizing sequence and applying Mazur's lemma. The minimizing function $u_{1}$ is a weak solution to the desired equation, that is

$$
\int_{\Omega}\left\langle d u_{1}, d \varphi\right\rangle d \mu=-\int_{\Omega} f \varphi d \mu
$$

for all $\varphi \in N_{0}^{1,2}(\Omega, \mu)$. From (3.9) it is immediate to see that $u_{1}$ is the desired solution; in addition, if in (3.9) we take $\varphi=u_{1}$, we have the Caccioppoli type estimate

$$
\left\|d u_{1}\right\|_{2} \leq c_{s}\|f\|_{2}
$$




\section{Functions of bounded variation and the perimeter measure}

The aim of this section is to study some properties of the perimeter measure of a ball in metric space. The properties we have in mind are needed in the characterization of a singular function which will be constructed in Section 6 .

Following [21], the definition of the total variation of a function $u \in L^{1}(X, \mu)$ is given by

$$
|D u|(X)=\inf \left\{\liminf _{j} \int_{X} g_{u_{j}} d \mu: u_{j} \in \operatorname{Lip}_{\mathrm{loc}}(X, \mu), u_{j} \rightarrow u \text { in } L_{\mathrm{loc}}^{1}(X, \mu)\right\} .
$$

A function $u$ is said to have bounded variation, that is, $u \in B V(X, \mu)$, if $|D u|(X)<\infty$. Moreover, a Borel set $E \subset X$ with finite measure is said to have finite perimeter if $\chi_{E} \in B V(X, \mu)$. We denote the perimeter measure of $E$ by $P(E, X)=\left|D \chi_{E}\right|(X)$.

To each function of bounded variation we associate a Borel regular measure, its total variation measure. This measure is defined on every open set $A \subset X$ using (4.1), that is,

$$
|D u|(A)=\inf \left\{\liminf _{j} \int_{A} g_{u_{j}} d \mu: u_{j} \in \operatorname{Lip}_{\mathrm{loc}}(A, \mu), u_{j} \rightarrow u \text { in } L_{\mathrm{loc}}^{1}(A, \mu)\right\} .
$$

We extend this measure to act on any Borel set $B \subset X$ by the Carathéodory construction

$$
|D u|(B)=\inf \{|D u|(A): A \text { open and } B \subset A\} ;
$$

for more details on this construction in the metric measure setting see Theorem 3.4 in $[21]$.

An equivalent definition can be also given by way of the Cheeger differentiable structure as follows:

$$
\left|D_{c} u\right|(X)=\inf \left\{\liminf _{j} \int_{X}\left|d u_{j}\right| d \mu: u_{j} \in \operatorname{Lip}_{\mathrm{loc}}(X, \mu), u_{j} \rightarrow u \text { in } L_{\mathrm{loc}}^{1}(X, \mu)\right\},
$$

and we shall say that $u$ has bounded total Cheeger variation if $\left|D_{c} u\right|(X)<\infty$; a set with Cheeger finite perimeter is a Borel set $E$ with finite measure such that $\left|D_{c} \chi_{E}\right|(X)<\infty$.

By the results contained in [7], it follows that these two definitions are equivalent, in the sense that $u$ has bounded total variation if and only if it has bounded total Cheeger variation. There exists a constant $c>1$ such that

$$
\frac{1}{c}|D u|(X) \leq\left|D_{c} u\right|(X) \leq c|D u|(X) .
$$

Also using the Cheeger differentiable structure, we have that $\left|D_{c} u\right|$ defines a finite Radon measure; the argument is similar to the case of $|D u|$ and so we refer to [21] for the proof.

A sequence of Lipschitz functions $\left(u_{j}\right)_{j}$ is said to converge in variation to a function $u \in B V(X, \mu)$ if $u_{j}$ converges to $u$ in $L_{\mathrm{loc}}^{1}(X, \mu)$ and

$$
\int_{X} g_{u_{j}} d \mu \rightarrow|D u|(X)
$$


The preceding definition of total variation does not specify the optimal sequence, i.e., the sequence which converges to $u$ in variation.

It is proved in Theorem 3.8 of [21] that the discrete convolution gives an approximation that is only comparable in variation with the optimal one. Note that for the optimal sequence, the vector valued measures $d \vec{\mu}_{j}=d u_{j} d \mu$, have uniformly bounded total variation. So, up to subsequences, they converge to some vector valued finite measure $\vec{\mu}_{\infty}$.

Remark 4.1. A sequence $\left(u_{j}\right)_{j}$ converging to $u$ in variation is optimal not only for the variation in $X$, but also for the variation in all open subsets $A$ with $|D u|(\partial A)=0$. In fact, by definition, we have that

$$
|D u|(A) \leq \liminf _{j \rightarrow \infty} \int_{A} g_{u_{j}} d \mu,
$$

but also that

$$
\begin{aligned}
|D u|(X \backslash \bar{A}) & \leq \limsup _{j \rightarrow \infty} \int_{X \backslash \bar{A}} g_{u_{j}} d \mu \leq \limsup _{j \rightarrow \infty}\left(\int_{X} g_{u_{j}} d \mu-\int_{A} g_{u_{j}} d \mu\right) \\
& =|D u|(X)-\liminf _{j \rightarrow \infty} \int_{A} g_{u_{j}} d \mu \leq|D u|(X \backslash A) .
\end{aligned}
$$

The preceding inequalities are indeed equalities if $|D u|(\partial A)=0$ and so $|D u|(X \backslash A)$ $=|D u|(X \backslash \bar{A})$. Hence the following two limits exist:

$$
\lim _{j \rightarrow \infty} \int_{X \backslash A} g_{u_{j}} d \mu=|D u|(X \backslash A),
$$

and

$$
\lim _{j \rightarrow \infty} \int_{A} g_{u_{j}} d \mu=|D u|(A) .
$$

An important tool in the theory of functions of bounded variation is the coarea formula. The version we work with in the present paper is a direct consequence of Proposition 4.2 in [21]. For any $u \in B V(X, \mu)$ and any Borel measurable function $f: X \rightarrow \mathbb{R}$, the following identities hold:

$$
\int_{X} f d|D u|=\int_{\mathbb{R}} \int_{X} f(x) d\left|D \chi_{E_{t}}\right|(x) d t
$$

and

$$
\int_{X} f d\left|D_{c} u\right|=\int_{\mathbb{R}} \int_{X} f(x) d\left|D_{c} \chi_{E_{t}}\right|(x) d t,
$$

where $E_{t}=\{u>t\}, t \in \mathbb{R}$, is the super-level set of $u$. We point out that in these formulae, due to the fact that the measures $|D u|$ and $\left|D_{c} u\right|$ are not absolutely continuous with respect to the measure $\mu$, it is important to consider the function $f$ and not an equivalent representative. Since the perimeter measure does not charge sets with zero 1-capacity, we can modify the function $f$ on such negligible sets. 
If $u$ is Lipschitz, (4.3) can be written as

$$
\int_{X} f g_{u} d \mu=\int_{\mathbb{R}} \int_{X} f d\left|D \chi_{E_{t}}\right|(x) d t .
$$

This follows by an argument contained in Theorem 6.2.2 of [6], and summarized in the following proposition. We will provide a proof here for the reader's convenience.

Proposition 4.2. Let $u \in \operatorname{Lip}(X)$. Then the total variation measure $d|D u|$ is given by $g_{u} d \mu$.

Proof. Lipschitz continuity of $u$ implies that $|D u|$ is absolutely continuous with respect to $\mu$ with density given by some function $G_{u}$. To see this, note that $u_{j}=u$ is a possible competitor in the definition of $|D u|$, and so for every open set $A \subset X$ (and hence for every set $A \subset X$ ) we have

$$
|D u|(A) \leq \int_{A} g_{u} d \mu
$$

Therefore the density function $G_{u} \leq g_{u} \mu$-a.e.

To prove the equality it suffices to prove that the function $G_{u}$ is an upper gradient of $u$. We take a sequence $\left(u_{j}\right)_{j}$ of Lipschitz functions converging to $u$ in $L^{1}(X, \mu)$ and with

$$
\lim _{j \rightarrow \infty} \int_{X} g_{u_{j}} d \mu=|D u|(X) .
$$

The sequence of measures $g_{u_{j}} d \mu$ is bounded, and so, up to a subsequence, it converges weakly to a measure $\mu_{\infty}$ that is still absolutely continuous with respect to $\mu$, i.e., $d \mu_{\infty}=g_{\infty} d \mu$. To see that $\mu_{\infty}$ is absolutely continuous with respect to $\mu$, it suffices to show that whenever $E \subset X$ is compact with $\mu(E)=0$, we have $\mu_{\infty}(E)=0$. To this end, we note that because $E$ is compact, for every $\varepsilon>0$ we can cover $E$ with a finite number of balls $B_{i}^{\varepsilon}$ with $\mu\left(\partial B_{i}^{\varepsilon}\right)=0$ so that the open set $A_{\varepsilon}=\cup_{i} B_{i}^{\varepsilon}$ contains $E$ and is such that $\mu\left(A_{\varepsilon}\right)<\varepsilon$ and $\mu\left(\partial A_{\varepsilon}\right)=0$. It follows that $|D u|\left(\partial A_{\varepsilon}\right)=0$ because of the absolute continuity of $|D u|$ established above.

Therefore, by Remark 4.1 we have

$$
\lim _{j \rightarrow \infty} \int_{A_{\varepsilon}} g_{u_{j}} d \mu=|D u|\left(A_{\varepsilon}\right) \leq \int_{A_{\varepsilon}} g_{u} d \mu \leq L \varepsilon,
$$

which implies that $\mu_{\infty}\left(A_{\varepsilon}\right) \leq L \varepsilon$. It follows that $\mu_{\infty}(E)=0$. For more general sets $E \subset X$ with $\mu(E)=0$, there is a Borel set $E_{0}$, containing $E$, such that $\mu\left(E_{0}\right)=0$. Because $\mu_{\infty}$ is a Borel measure, $\mu_{\infty}\left(E_{0}\right)$ is the supremum of all $\mu_{\infty}(K)$, the supremum taken over all compact sets $K \subset E_{0}$. Given that $\mu(K)=0$ and so $\mu_{\infty}(K)=0$, it follows that $\mu_{\infty}\left(E_{0}\right)=0$ and so $\mu_{\infty}(E)=0$. Then $g_{u_{j}}$ converges to $g_{\infty}$ weakly in $L^{1}(X, \mu)$.

To summarize, we have $u_{j} \rightarrow u$ in $L^{1}(X)$ and $g_{u_{j}} \rightarrow g_{\infty}$ weakly in $L^{1}(X)$. Now, an invocation of the Mazur lemma, together with Lemma 3.1 in [16], shows that $g_{\infty}$ is a weak upper gradient of $u$; it follows that $g_{u} \leq g_{\infty}$ a.e. in $X$. 
Given that balls have finite $\mu$-measure, when $x \in X$, for almost every $r>0$ we have $\mu\left(\partial B_{r}(x)\right)=0$. For such $r>0$, by (4.2) with $A=B_{r}(x)$,

$$
|D u|\left(B_{r}(x)\right) \leq \int_{B_{r}(x)} g_{\infty} d \mu \leq \liminf _{j \rightarrow \infty} \int_{B_{r}(x)} g_{u_{j}} d \mu=|D u|\left(B_{r}(x)\right)=\int_{B_{r}(x)} G_{u} d \mu,
$$

and so we get $g_{\infty}(x)=G_{u}(x)$ for $x \in X$ that are Lebesgue points for both $G_{u}$ and $g_{\infty}$. It follows that $G_{u} \leq g_{u} \leq g_{\infty}=G_{u}$ a.e. in $X$, and the claim follows.

From now on, we assume that $X$ is also a geodesic space. This is not an overly restrictive assumption, since $X$, by the virtue of supporting a Poincaré inequality and being complete, is a quasiconvex space. It follows that in our setting, a biLipschitz change in the metric does result in a geodesic space.

Let us fix a point $x_{0} \in X$. Since $X$ is assumed to be a geodesic space, by the results in [7], the function $u_{x_{0}}(x)=d\left(x, x_{0}\right)$ is $\operatorname{Lipschitz}$ with $\operatorname{Lip}\left(u_{x_{0}}\right)=g_{u_{x_{0}}} \equiv 1$. Moreover, we may write $B_{t}\left(x_{0}\right)=\left\{u_{x_{0}}<t\right\}$ and so by the coarea formula (4.3) we obtain for any positive $r>0$ that

$$
\int_{0}^{r} P\left(B_{t}\left(x_{0}\right), X\right) d t=\mu\left(B_{r}\left(x_{0}\right)\right)<\infty .
$$

Thus the map $t \mapsto P\left(B_{t}\left(x_{0}\right), X\right)$ is a measurable locally integrable function. This implies that for almost every $r>0$,

$$
\begin{aligned}
P\left(B_{r}\left(x_{0}\right), X\right) & =\lim _{\varepsilon \rightarrow 0} \frac{1}{\varepsilon} \int_{r-\varepsilon}^{r} P\left(B_{t}\left(x_{0}\right), X\right) d t=\lim _{\varepsilon \rightarrow 0} \frac{1}{\varepsilon} \int_{r}^{r+\varepsilon} P\left(B_{t}\left(x_{0}\right), X\right) d t \\
& =\lim _{\varepsilon \rightarrow 0} \frac{1}{2 \varepsilon} \int_{r-\varepsilon}^{r+\varepsilon} P\left(B_{t}\left(x_{0}\right), X\right) d t .
\end{aligned}
$$

In particular, for almost every $r>0$ the perimeter measure coincides with the Minkowski content

$$
P\left(B_{r}\left(x_{0}\right), X\right)=\lim _{\varepsilon \rightarrow 0} \frac{\mu\left(B_{r}\left(x_{0}\right)\right)-\mu\left(B_{r-\varepsilon}\left(x_{0}\right)\right)}{\varepsilon} .
$$

For a ball $B_{r}\left(x_{0}\right)$ satisfying (4.4), we can consider the sequence of functions $\left(u_{\varepsilon}\right)_{\varepsilon>0}$, where

$$
u_{\varepsilon}(x)=\max \left\{\min \left\{\frac{r-d\left(x_{0}, x\right)}{\varepsilon}, 1\right\}, 0\right\}=\min \left\{\frac{1}{\varepsilon} d\left(x, X \backslash B_{r}\left(x_{0}\right)\right), 1\right\} .
$$

For a such function $u_{\varepsilon}$, we have that $g_{u_{\varepsilon}}=\frac{1}{\varepsilon} \chi_{B_{r}\left(x_{0}\right) \backslash B_{r-\varepsilon}\left(x_{0}\right)}$ and

$$
\int_{X} g_{u_{\varepsilon}} d \mu=\frac{1}{\varepsilon} \int_{B_{r}\left(x_{0}\right) \backslash B_{r-\varepsilon}\left(x_{0}\right)} d \mu \rightarrow P\left(B_{r}\left(x_{0}\right), X\right),
$$

that is, the sequence $u_{\varepsilon}$ converges to $\chi_{B_{r}\left(x_{0}\right)}$ in variation. This also means that the sequence of vector valued measures $\left(\left|d u_{\varepsilon}\right| d \mu\right)_{\varepsilon}$ is equibounded:

$$
\left|D_{c} u_{\varepsilon}\right|(X)=\int_{X}\left|d u_{\varepsilon}\right| d \mu \leq c<\infty
$$


for some positive constant $c$. Therefore there exists a subsequence $\varepsilon_{j} \rightarrow 0$ such that, setting $u_{j}=u_{\varepsilon_{j}}$, the sequence of vector-valued measures $d u_{j} d \mu$ is weakly convergent to some vector valued measure $\vec{\mu}_{\infty}$. This measure is absolutely continuous with respect to both $\left|D_{c} \chi_{B_{r}\left(x_{0}\right)}\right|$ and $\left|D \chi_{B_{r}\left(x_{0}\right)}\right|$. Indeed, if, for instance, $\left|D_{c} \chi_{B_{r}\left(x_{0}\right)}\right|(E)=0$, where $E \subset X$ is compact, we can find for every $\varepsilon>0$ an open set $A_{\varepsilon} \supset E$ such that $\left|D_{c} \chi_{B_{r}\left(x_{0}\right)}\right|\left(\partial A_{\varepsilon}\right)=0$ and $\left|D_{c} \chi_{B_{r}\left(x_{0}\right)}\right|\left(A_{\varepsilon}\right)<\varepsilon$. Reasoning as in the proof of Proposition 4.2 we may conclude that

$$
\left|\vec{\mu}_{\infty}\right|(E) \leq\left|\vec{\mu}_{\infty}\right|\left(A_{\varepsilon}\right) \leq \liminf _{j \rightarrow \infty} \int_{A_{\varepsilon}}\left|d u_{j}\right| d \mu=\left|D_{c} \chi_{B_{r}\left(x_{0}\right)}\right|\left(A_{\varepsilon}\right)<\varepsilon .
$$

We may hence write

$$
\vec{\mu}_{\infty}=\nu_{x_{0}, r}\left|D_{c} \chi_{B_{r}\left(x_{0}\right)}\right|=\sigma_{x_{0}, r}\left|D \chi_{B_{r}\left(x_{0}\right)}\right|
$$

for some vector-valued $\left|D_{c} \chi_{B_{r}\left(x_{0}\right)}\right|$-measurable function $\nu_{x_{0}, r}$ and $\left|D \chi_{B_{r}\left(x_{0}\right)}\right|$-measurable function $\sigma_{x_{0}, r}$. In particular, the function $\sigma_{x_{0}, r}$ plays the role of the normal vector at the boundary of $B_{r}\left(x_{0}\right)$. In this context, it is not clear from the definition if it is a unit vector. For the sake of simplicity, if no confusion may arise, we simply denote the functions $\nu_{x_{0}, r}$ and $\sigma_{x_{0}, r}$ by $\nu$ and $\sigma$, respectively.

We can summarize the previous construction in the following definition.

Definition 4.3. We shall call a ball $B_{r}\left(x_{0}\right)$ regular if the equation (4.4) is valid and if there exists a sequence $\varepsilon_{j} \rightarrow 0$ such that for the sequence of functions $u_{j}=u_{\varepsilon_{j}}$, referred to as an optimal sequence and defined in (4.5), the following hold true:

(1) $g_{u_{j}} d \mu$ converges weakly to $d\left|D \chi_{B_{r}\left(x_{0}\right)}\right|$;

(2) $d u_{j} d \mu$ converges weakly to $\sigma d\left|D \chi_{B_{r}\left(x_{0}\right)}\right|$ for some $\left|D \chi_{B_{r}\left(x_{0}\right)}\right|$-measurable vector-valued function $\sigma=\sigma_{x_{0}, r}$.

Almost every ball is regular in the sense that for every $x_{0} \in X$ and for almost every $r>0$ the ball $B_{r}\left(x_{0}\right)$ is regular. However, the vector $\sigma$ is not a priori unique and it is not clear whether it depends on the sequence $\varepsilon_{j}$ we consider.

The given notion of regularity relates to interior regularity of a ball. One can also consider the notions of outer and two-sided regularity and obtain that for almost every radius $r>0$ the ball $B_{r}\left(x_{0}\right)$ has inner, outer, and two-sided regularity.

\section{Divergence measures and Gauss-Green formulas}

Here we consider divergence-measure fields, i.e., a class of vector fields $\vec{F}: X \rightarrow \mathbb{R}^{k}$ belonging to the space $L^{2}\left(\Omega, \mathbb{R}^{k}, \mu\right)$ and for which $\operatorname{div} \vec{F}$ is a measure. In the metric space framework of the present paper, we generalize some results obtained by Ziemer in [27].

Previously, Thompson and Thompson in [26] constructed a divergence form in the setting of Minkowski spaces, and they proved a Minkowski space analogue of the Gauss-Green theorem. 
The aim of this section is to study the operator $\operatorname{div}$ on $\operatorname{Lip}_{c}(\Omega)$ with values in the space of measures, that is, we want to define for $\vec{F} \in L^{2}\left(\Omega, \mathbb{R}^{k}, \mu\right)$ the distribution

$$
\langle u, \operatorname{div} \vec{F}\rangle:=-\int_{\Omega}\langle\vec{F}, d u\rangle d \mu,
$$

for $u \in \operatorname{Lip}_{c}(\Omega)$. In the following we adopt the notation from [8] and [27].

Definition 5.1. We say that $\vec{F} \in L^{2}\left(\Omega, \mathbb{R}^{k}, \mu\right)$ is in the class $\mathcal{D} \mathcal{M}^{2}(\Omega)$ if there is a signed finite Radon measure, denoted by $\operatorname{div} \vec{F} \in \mathscr{M}_{b}(\Omega)$, on $\Omega$ such that

$$
\int_{\Omega} u d \operatorname{div} \vec{F}=-\int_{\Omega}\langle\vec{F}, d u\rangle d \mu \quad \text { for all } u \in \operatorname{Lip}_{c}(\Omega) .
$$

Remark 5.2. By an argument similar to the proof of Lemma 3.6, we can prove that if $\vec{F} \in \mathcal{D} \mathcal{M}^{2}(\Omega)$, then the measure $\operatorname{div} \vec{F}$ does not charge sets with zero 2-capacity. Therefore, condition (5.2) can be extended to any $u \in N_{0}^{1,2}(\Omega, \mu) \cap L^{\infty}(\Omega, \mu)$.

Note that when $\vec{F} \in L^{2}\left(\Omega, \mathbb{R}^{k}, \mu\right)$ the operator $T_{\vec{F}}: N_{0}^{1,2}(\Omega, \mu) \rightarrow \mathbb{R}$ given by

$$
T_{\vec{F}}(u)=\int_{\Omega}\langle\vec{F}, d u\rangle d \mu
$$

is a bounded linear operator on the Hilbert space $\left(N_{0}^{1,2}(\Omega, \mu), \mathcal{E}_{1}\right)$. Therefore, by the Riesz representation theorem, there exists a function $v \in N_{0}^{1,2}(\Omega, \mu)$ such that whenever $u \in N_{0}^{1,2}(\Omega, \mu), T_{\vec{F}}(u)=\mathcal{E}_{1}(v, u)$. Hence, if $\vec{F} \in \mathcal{D} \mathcal{M}^{2}(\Omega)$, then for all $u \in N_{0}^{1,2}(\Omega, \mu)$ we obtain

$$
\int_{\Omega}\langle\vec{F}, d u\rangle d \mu=\int_{\Omega} u v d \mu+\int_{\Omega}\langle d u, d v\rangle d \mu,
$$

that is,

$$
\int_{\Omega} u d \operatorname{div} \vec{F}+\int_{\Omega} u v d \mu=-\int_{\Omega}\langle d u, d v\rangle d \mu .
$$

It follows that $v \in \operatorname{Dom}(\mathscr{D} \Omega)$ with

$$
d \mathscr{D}_{\Omega} v=-v d \mu-d \operatorname{div} \vec{F} .
$$

This proves the following lemma.

Lemma 5.3. Given a domain $\Omega \subset X$, a map $\vec{F} \in L^{2}\left(\Omega, \mathbb{R}^{k}, \mu\right)$ is in the class $\mathcal{D M}^{2}(\Omega)$ if and only if there exists $v \in \operatorname{Dom}(\mathscr{D} \Omega)$ such that

$$
d \mathscr{D}_{\Omega} v=-v d \mu-d \operatorname{div} \vec{F}
$$

in the sense of distributions on $N_{0}^{1,2}(\Omega, \mu)$.

We can also state the following simple properties of the divergence measure. 
Lemma 5.4. Let $\vec{F} \in \mathcal{D} \mathcal{M}^{2}(\Omega)$. Then $\operatorname{spt}(\operatorname{div} \vec{F}) \subset \operatorname{spt}(\vec{F})$. Moreover, if $v \in$ $\operatorname{Dom}\left(\mathscr{D}_{\Omega}\right)$, then $d v \in \mathcal{D} \mathcal{M}^{2}(\Omega)$ with $\operatorname{div} d v=\mathscr{D} \Omega v$.

Proof. The first statement follows by considering $A=\Omega \backslash \operatorname{spt}(\vec{F})$, so we have that

$$
\begin{aligned}
|\operatorname{div} \vec{F}|(A) & =\sup \left\{\int_{A} \varphi d \operatorname{div} \vec{F}: \varphi \in \operatorname{Lip}_{c}(A),\|\varphi\|_{\infty} \leq 1\right\} \\
& =\sup \left\{\int_{A}\langle\vec{F}, d \varphi\rangle d \mu: \varphi \in \operatorname{Lip}_{c}(A),\|\varphi\|_{\infty} \leq 1\right\}=0 .
\end{aligned}
$$

For the second part, let $v \in \operatorname{Dom}\left(\mathscr{D}_{\Omega}\right)$. Then there exists a signed finite Radon measure $\mathscr{D}_{\Omega} v \in \mathscr{M}_{b}(X)$ such that

$$
\int_{\Omega}\langle d v, d u\rangle d \mu=-\int_{\Omega} u d \mathscr{D} \Omega v
$$

for all $u \in N_{0}^{1,2}(\Omega, \mu) \cap L^{\infty}(\Omega, \mu)$. From this and by Remark 5.2, we may conclude that $d v \in \mathcal{D M}^{2}(\Omega)$ with $\operatorname{div} d v=\mathscr{D} \Omega v$, and the claim follows.

We now state the following two propositions on the Gauss-Green type integration by parts formula for vector fields in $\mathcal{D} \mathcal{M}^{\infty}(\Omega)$, that is for vector fields $\vec{F}$ in $L^{\infty}\left(\Omega, \mathbb{R}^{k}, \mu\right) \cap \mathcal{D} \mathcal{M}^{2}(\Omega)$.

Proposition 5.5. Let $\vec{F} \in \mathcal{D} \mathcal{M}^{\infty}(\Omega) \cap C\left(\Omega, \mathbb{R}^{k}\right)$ and $B_{r}\left(x_{0}\right) \subset \Omega$ be a regular ball. The following Gauss-Green formula

$$
\int_{B_{r}\left(x_{0}\right)} f d \operatorname{div} \vec{F}+\int_{B_{r}\left(x_{0}\right)}\langle\vec{F}, d f\rangle d \mu=-\int_{\Omega} f\left\langle\vec{F}, \sigma_{x_{0}, r}\right\rangle d\left|D \chi_{B_{r}\left(x_{0}\right)}\right|,
$$

holds for all $f \in \operatorname{Lip}_{c}(\Omega)$. If the support of $\vec{F}$ is disjoint from $\partial B_{r}\left(x_{0}\right)$, then the requirement that $\vec{F}$ is continuous can be removed.

Proof. We can consider an optimal sequence of locally Lipschitz functions $\left(u_{j}\right)_{j}$ converging to $\chi_{B_{r}\left(x_{0}\right)}$ in variation as in (4.5). Then we have by the Leibniz rule that

$$
\int_{\Omega} u_{j} f d \operatorname{div} \vec{F}=-\int_{\Omega}\left\langle\vec{F}, d\left(u_{j} f\right)\right\rangle d \mu=-\int_{\Omega} u_{j}\langle\vec{F}, d f\rangle d \mu-\int_{\Omega} f\left\langle\vec{F}, d u_{j}\right\rangle d \mu .
$$

We notice that

$$
\left|\int_{\Omega} u_{j} f d \operatorname{div} \vec{F}-\int_{B_{r}\left(x_{0}\right)} f d \operatorname{div} \vec{F}\right| \leq\|f\|_{\infty}|\operatorname{div} \vec{F}|\left(B_{r}\left(x_{0}\right) \backslash B_{r-\varepsilon_{j}}\left(x_{0}\right)\right),
$$

and that the right-hand side of (5.3) tends to 0 as $j \rightarrow \infty$. Thus we may conclude that

$$
\lim _{j \rightarrow \infty} \int_{\Omega} u_{j} f d \operatorname{div} \vec{F}=\int_{B_{r}\left(x_{0}\right)} f d \operatorname{div} \vec{F}
$$


Also, by the fact that both $\vec{F}$ and $d f$ are in $L^{\infty}(\Omega)$, by an application of the Lebesgue dominated convergence theorem we obtain

$$
\lim _{j \rightarrow \infty} \int_{\Omega} u_{j}\langle\vec{F}, d f\rangle d \mu=\int_{B_{r}\left(x_{0}\right)}\langle\vec{F}, d f\rangle d \mu
$$

We also have, due to the continuity of $\vec{F}$, that

$$
\lim _{j \rightarrow \infty} \int_{\Omega} f\left\langle\vec{F}, d u_{j}\right\rangle d \mu=\int_{\Omega} f\left\langle\vec{F}, \sigma_{x_{0}, r}\right\rangle d\left|D \chi_{B_{r}\left(x_{0}\right)}\right|,
$$

and so the proof is completed.

Remark 5.6. We point out that property (5.3) is a consequence of the choice of an optimal sequence $\left(u_{j}\right)_{j}$ to be an inner approximation of the characteristic function $\chi_{B_{r}\left(x_{0}\right)}$. If we chose, for instance, an outer approximation, then the preceding integration by parts formula would be as follows:

$$
\int_{\bar{B}_{r}\left(x_{0}\right)} f d \operatorname{div} \vec{F}+\int_{B_{r}\left(x_{0}\right)}\langle\vec{F}, d f\rangle d \mu=-\int_{\Omega} f\left\langle\vec{F}, \tilde{\sigma}_{x_{0}, r}\right\rangle d\left|D \chi_{B_{r}\left(x_{0}\right)}\right|,
$$

for all $f \in \operatorname{Lip}_{c}(\Omega)$, where $\tilde{\sigma}_{x_{0}, r}$ is the density of the vector-valued measure obtained as a weak limit by way of the gradients of this new sequence as in Definition 4.3.

We also point out that the previous proposition can be extend to more general sets $E \subset \Omega$ with finite perimeter whenever a Minkowski content characterization of the perimeter, analogous to (4.4), holds. In this case, the boundary of $E$ has to be considered as the essential, or the measure-theoretic, boundary of $E$, i.e., the set of all points at which the density of $E$ is neither 0 nor 1 .

We prove the following main theorem of this section, which is a generalization of Proposition 5.5, without requiring continuity of the vector field. This theorem should be thought of as the generalization of the Gauss-Green theorem of the Euclidean setting.

Theorem 5.7. Let $\vec{F} \in \mathcal{D} \mathcal{M}^{\infty}(\Omega)$ and let $B_{r}\left(x_{0}\right) \subset \Omega$ be a regular ball. Then the following extended Gauss-Green formula

$$
\int_{B_{r}\left(x_{0}\right)} f d \operatorname{div} \vec{F}+\int_{B_{r}\left(x_{0}\right)}\langle\vec{F}, d f\rangle d \mu=\int_{\Omega} f(\vec{F} \cdot \nu)_{\partial B_{r}\left(x_{0}\right)}^{-} d\left|D \chi_{B_{r}\left(x_{0}\right)}\right|,
$$

holds for all $f \in N^{1,2}(\Omega, \mu) \cap L^{\infty}(\Omega, \mu)$, where $(\vec{F} \cdot \nu)_{\partial B_{r}\left(x_{0}\right)}^{-}$is the interior normal trace of $\vec{F}$ on $\partial B_{r}\left(x_{0}\right)$.

Proof. We use the optimal sequence $\left(u_{j}\right)_{j}$ defined in (4.5). Then, as in the proof of Proposition 5.5, by the definition of $\operatorname{div} \vec{F}$ (Definition 5.1) and the Lebesgue 
dominated convergence theorem

$$
\begin{aligned}
\lim _{j \rightarrow \infty} \int_{\Omega} f\left\langle\vec{F}, d u_{j}\right\rangle d \mu & =\lim _{j \rightarrow \infty}\left(\int_{\Omega}\left\langle\vec{F}, d\left(u_{j} f\right)\right\rangle d \mu-\int_{\Omega} u_{j}\langle\vec{F}, d f\rangle d \mu\right) \\
& =-\lim _{j \rightarrow \infty}\left(\int_{\Omega} u_{j} f d \operatorname{div} \vec{F}+\int_{\Omega} u_{j}\langle\vec{F}, d f\rangle d \mu\right) \\
& =-\int_{B_{r}\left(x_{0}\right)} f d \operatorname{div} \vec{F}-\int_{B_{r}\left(x_{0}\right)}\langle\vec{F}, d f\rangle d \mu .
\end{aligned}
$$

For the sequence $\left(L_{j}\right)_{j}$ of operators given by

$$
L_{j}(f):=\int_{\Omega} f\left\langle\vec{F}, d u_{j}\right\rangle d \mu,
$$

we have that $\left|L_{j}(f)\right| \leq C\|\vec{F}\|_{\infty}\|f\|_{\infty}$, where the positive constant $C$ is given by

$$
C=\sup _{j \in \mathbb{N}} \int_{\Omega}\left|d u_{j}\right| d \mu<\infty .
$$

Indeed, $C$ is finite since the ball $B_{r}\left(x_{0}\right)$ has finite perimeter. In particular, $C$ is independent of both $f$ and $\vec{F}$ and so, by the above argument, the operator

$$
L(f):=\lim _{j \rightarrow \infty} L_{j}(f)
$$

is bounded over $\operatorname{Lip}_{c}(\Omega)$ and admits an extension to $C_{c}(\Omega)$. This in turn implies that there exists a measure $\nu \in \mathscr{M}_{b}(\Omega)$ such that, for any $f \in C_{c}(\Omega)$,

$$
L(f)=\int_{\Omega} f d \nu
$$

The measure $\nu$ is concentrated on $\partial B_{r}\left(x_{0}\right)$; in fact take any compact set $K$ such that $K \cap \partial B_{r}\left(x_{0}\right)=\emptyset$, an open set $A \supset K \operatorname{such}$ that $\operatorname{dist}\left(A, \partial B_{r}\left(x_{0}\right)\right)>0$, and take $\varepsilon_{j}<\operatorname{dist}\left(A, \partial B_{r}\left(x_{0}\right)\right)$. Then, since $\operatorname{spt}\left(d u_{j}\right) \cap A=\emptyset$, we obtain for any $f \in \operatorname{Lip}_{c}(A)$,

$$
\int_{\Omega} f d \nu=\lim _{j \rightarrow \infty} \int_{\Omega} f\left\langle\vec{F}, d u_{j}\right\rangle d \mu=0
$$

that is $|\nu|(K)=|\nu|(A)=0$. This property extends to any Borel set $E$ such that $E \cap \partial B_{r}\left(x_{0}\right)=\emptyset$ since

$$
|\nu|(E)=\sup _{K \subset E}|\nu|(K)=0 .
$$

Also $\nu$ can be seen to be absolutely continuous with respect to $\left|D_{c} \chi_{B_{r}\left(x_{0}\right)}\right|$; indeed, if $E$ is a Borel set such that $\left|D_{c} \chi_{B_{r}\left(x_{0}\right)}\right|(E)=0$, then there exists an open set $A_{\varepsilon}$ such that $\left|D_{c} \chi_{B_{r}\left(x_{0}\right)}\right|\left(A_{\varepsilon}\right)<\varepsilon$. Fix a compact set $K \subset E$ and an open set $A \supset K$ such that $\bar{A} \subset A_{\varepsilon}$. Then, for any $f \in \operatorname{Lip}_{c}(A)$ with $\|f\|_{\infty} \leq 1$ we have that

$$
\left|\int_{A} f\left\langle\vec{F}, d u_{j}\right\rangle d \mu\right| \leq \limsup _{j \rightarrow \infty}\|\vec{F}\|_{\infty} \int_{A}\left|d u_{j}\right| d \mu \leq\|\vec{F}\|_{\infty}\left|D_{c} \chi_{B_{r}\left(x_{0}\right)}\right|(\bar{A})<\varepsilon\|\vec{F}\|_{\infty},
$$

that is $|\nu|(A)<\varepsilon$. Therefore, since $\varepsilon$ is arbitrary, $|\nu|(K)=0$. Finally, by taking the supremum over $K \subset E$, we obtain that $|\nu|(E)=0$, and hence $\nu$ is absolutely continuous with respect to $\left|D_{c} \chi_{B_{r}\left(x_{0}\right)}\right|$. 
To conclude, there exists $(\vec{F} \cdot \nu)_{\partial B_{r}\left(x_{0}\right)}^{-} \in L^{1}\left(\left|D \chi_{B_{r}\left(x_{0}\right)}\right|\right)$ such that

$$
L(f)=-\int_{\Omega} f(\vec{F} \cdot \nu)_{\partial B_{r}\left(x_{0}\right)}^{-} d\left|D \chi_{B_{r}\left(x_{0}\right)}\right| .
$$

This map defines, in the metric setting, the interior normal trace of $\vec{F}$ on $\partial B_{r}\left(x_{0}\right)$, and the integration by parts formula (5.4) holds.

Remark 5.8. The term interior normal trace can be justified by the following facts. If $\vec{F} \in \mathcal{D} \mathcal{M}^{\infty}(\Omega) \cap C\left(\Omega, \mathbb{R}^{k}\right)$, then by Proposition 5.5 we get that

$$
(\vec{F} \cdot \nu)_{\partial B_{r}\left(x_{0}\right)}^{-}=-\left\langle\vec{F}, \sigma_{x_{0}, r}\right\rangle .
$$

In addition, also when $\vec{F}$ is not continuous, recalling that with $u_{x_{0}}(x)=d\left(x, x_{0}\right)$,

$$
d u_{j}(x)=-\frac{1}{\varepsilon_{j}} d u_{x_{0}}(x) \chi_{B_{r}\left(x_{0}\right) \backslash B_{r-\varepsilon_{j}}\left(x_{0}\right)}(x),
$$

and by using the coarea formula (4.3), we can write

$$
\begin{aligned}
\int_{\Omega} f\left\langle\vec{F}, d u_{j}\right\rangle d \mu & =-\frac{1}{\varepsilon_{j}} \int_{B_{r}\left(x_{0}\right) \backslash B_{r-\varepsilon_{j}}\left(x_{0}\right)} f\left\langle\vec{F}, d u_{x_{0}}\right\rangle d \mu \\
& =-\frac{1}{\varepsilon_{j}} \int_{r-\varepsilon_{j}}^{r} \int_{\Omega} f\left\langle\vec{F}, d u_{x_{0}}\right\rangle d\left|D \chi_{B_{t}\left(x_{0}\right)}\right| d t
\end{aligned}
$$

Therefore, we have obtained that

$$
\int_{\Omega} f(\vec{F} \cdot \nu)_{\partial B_{r}\left(x_{0}\right)}^{-} d\left|D \chi_{B_{r}\left(x_{0}\right)}\right|=-\lim _{j \rightarrow \infty} \frac{1}{\varepsilon_{j}} \int_{r-\varepsilon_{j}}^{r} \int_{\Omega} f\left\langle\vec{F}, d u_{x_{0}}\right\rangle d\left|D \chi_{B_{t}\left(x_{0}\right)}\right| d t
$$

which gives meaning to the following equality in terms of the trace:

$$
\int_{\Omega} f(\vec{F} \cdot \nu)_{\partial B_{r}\left(x_{0}\right)}^{-} d\left|D \chi_{B_{r}\left(x_{0}\right)}\right|=-\int_{\Omega} f\left\langle\vec{F}, d u_{x_{0}}\right\rangle d\left|D \chi_{B_{r}\left(x_{0}\right)}\right|,
$$

and to the fact that the vector $d u_{x_{0}}$ defines in a weak sense the normal vector $\sigma_{x_{0}, r}$ to $\partial B_{r}\left(x_{0}\right)$.

Remark 5.9. Observe that in the proof of Proposition 5.7 we have used a particular optimal sequence. It turns out, nevertheless, that the interior normal trace $(\vec{F} \cdot \nu)_{\partial B_{r}\left(x_{0}\right)}^{-}$does not depend on this particular choice. This fact is a direct consequence of equation (5.4), since then formula

$$
\int_{\Omega} f(\vec{F} \cdot \nu)_{\bar{\partial} B_{r}\left(x_{0}\right)}^{-} d\left|D \chi_{B_{r}\left(x_{0}\right)}\right|=\int_{B_{r}\left(x_{0}\right)} f d \operatorname{div} \vec{F}+\int_{B_{r}\left(x_{0}\right)}\langle\vec{F}, d f\rangle d \mu
$$

uniquely identifies the values of $(\vec{F} \cdot \nu)_{\partial B_{r}\left(x_{0}\right)}^{-}$. 
Remark 5.10. By Theorem 5.3 in [2] (see also [3]), formula (5.4) can also be written as

$$
\int_{B_{r}\left(x_{0}\right)} f d \operatorname{div} \vec{F}+\int_{B_{r}\left(x_{0}\right)}\langle\vec{F}, d f\rangle d \mu=\int_{\partial^{*} B_{r}\left(x_{0}\right)} f(\vec{F} \cdot \nu)_{\partial B_{r}\left(x_{0}\right)}^{-} \vartheta_{x_{0}, r} d \mathcal{S}^{h},
$$

where $\partial^{*} B_{r}\left(x_{0}\right)$ is the essential boundary of $B_{r}\left(x_{0}\right), \mathcal{S}^{h}$ is the spherical Hausdorff measure defined using the Carathéodory construction based on the gauge function

$$
h\left(\bar{B}_{\varrho}\right)=\frac{\mu\left(\bar{B}_{\varrho}\right)}{\varrho},
$$

and $\vartheta_{x_{0}, r}: X \rightarrow\left[c, c_{d}\right]$ is a Borel function depending, in general, on the ball $B_{r}\left(x_{0}\right)$, and $c$ is a positive constant and $c_{d}$ the doubling constant of $\mu$.

\section{Harmonicity and the mean value property}

In this section, we shall follow the approach of [15] and construct, for any regular ball $B_{r}\left(x_{0}\right) \subset X$ and any $\bar{x} \in B_{r}\left(x_{0}\right)$ the Green function on $B_{r}\left(x_{0}\right)$ with singularity at $\bar{x}$, that is an extended real-valued function $G(x)=G_{B_{r}\left(x_{0}\right)}^{\bar{x}}(x)$ such that

1) $G$ is strictly positive and harmonic in $B_{r}\left(x_{0}\right) \backslash\{\bar{x}\}$;

2) $G \in N^{1,2}\left(X \backslash B_{\varepsilon}(\bar{x})\right)$ for any $\varepsilon>0$ and $\left.G\right|_{X \backslash \bar{B}_{r}\left(x_{0}\right)}=0$;

3) for every $y \in \partial B_{r}\left(x_{0}\right)$,

$$
\lim _{x \rightarrow y} G(x)=0
$$

4) $G$ is singular at $\bar{x}$; that is,

$$
\lim _{x \rightarrow \bar{x}} G(x)=\infty
$$

5) for all $0<a \leq b$,

$$
\mathrm{Cap}_{2}\left(\left\{x \in B_{r}\left(x_{0}\right): G(x) \geq b\right\},\left\{x \in B_{r}\left(x_{0}\right): G(x)>a\right\}\right)=\frac{1}{b-a} .
$$

In [15] the authors constructed the Green function of a relatively compact domain with the aforementioned properties in metric measure spaces; we refer also to [14] and [9]. We can state the existence and main properties of the Green function in the following theorem. We assume that $X$ supports a $(1,2)$-Poincaré inequality.

Theorem 6.1. Let $\Omega \subset X$ be a relatively compact domain. Then there exists the Green function $G=G_{\Omega}^{\bar{x}}$ with singularity at $\bar{x} \in \Omega$. In addition, $d G \in L^{2}\left(X \backslash B_{\varepsilon}(\bar{x})\right)$ for any $\varepsilon>0$ and

$$
\mathscr{D}_{X \backslash B_{\varepsilon}(\bar{x})} G=-\nu_{\Omega}^{G},
$$

where $\nu_{\Omega}^{G}$ is a positive Radon measure in the dual $N_{0}^{1,2}\left(X \backslash B_{\varepsilon}(\bar{x})\right)^{*}$ concentrated on $\partial \Omega$. 
Moreover, $G$ admits the measure-valued Laplace operator

$$
\mathscr{D}_{X} G=\delta_{\bar{x}}-\nu_{\Omega}^{G},
$$

in the sense that, for any $v \in N^{1,2}(X)$ continuous at $\bar{x}$, then

$$
\int_{X}\langle d G, d v\rangle d \mu=\int_{\partial \Omega} v d \nu_{\Omega}^{G}-v(\bar{x}) .
$$

Proof. We refer to [15] for the details on the construction of $G$. We sketch the main steps needed in the definition. We find a harmonic function on $\Omega \backslash \bar{B}_{\varepsilon_{j}}(\bar{x})$

$$
G_{j}=\frac{v_{j}}{\operatorname{Cap}_{2}\left(\bar{B}_{\varepsilon_{j}}(\bar{x}), \Omega\right)},
$$

where $B_{\varepsilon_{j}}(\bar{x})$ is a regular ball, $\varepsilon_{j} \searrow 0, \varepsilon_{j}<\operatorname{dist}(\bar{x}, \partial \Omega)$, and $v_{j}$ is the potential of $\bar{B}_{\varepsilon_{j}}(\bar{x})$ with respect to $\Omega$; that is $v_{j} \in N^{1,2}(X)$ is harmonic in $\Omega \backslash \bar{B}_{\varepsilon_{j}}(\bar{x}), v_{j}=0$ on $X \backslash \Omega$ and $v_{j}=1$ on $\bar{B}_{\varepsilon_{j}}(\bar{x})$. It is then shown that, up to subsequences, the functions $\left(G_{j}\right)_{j}$ converge locally uniformly in $X \backslash\{\bar{x}\}$ to a function $G$. The limit function $G$ has the desired properties of a Green function.

Let us fix a positive sequence $\left(M_{i}\right)_{i \geq 0}$ such that $M_{i} \nearrow \infty$, and the truncations

$$
T_{i} G:=\min \left\{G, M_{i}\right\} .
$$

There exists a sequence $r_{i} \searrow 0$ of radii such that

$$
E_{i} \subset B_{r_{i}}(\bar{x}),
$$

where we have written $E_{i}=\left\{x \in \Omega: G(x)>M_{i}\right\}$; and we may consider the case in which $r_{i}<\varepsilon$. Then $T_{i} G=G$ on $X \backslash B_{\varepsilon}(\bar{x})$ and $T_{i} G$ is subharmonic in $X \backslash B_{\varepsilon}(\bar{x})$. By [5] (we refer also to [20] for a detailed description in the Euclidean case) there exists a positive Radon measure $\nu_{\Omega}^{G}$ in the dual $N_{0}^{1,2}\left(X \backslash B_{\varepsilon}(\bar{x})\right)^{*}$ such that for all $v \in \operatorname{Lip}_{c}\left(X \backslash B_{\varepsilon}(\bar{x})\right)$ we have

$$
\int_{X \backslash B_{\varepsilon}(\bar{x})}\langle d G, d v\rangle d \mu=\int_{X \backslash B_{\varepsilon}(\bar{x})} v d \nu_{\Omega}^{G} .
$$

If $v \in \operatorname{Lip}_{c}(X \backslash \bar{\Omega})$, the fact that $G=0$ on $X \backslash \bar{\Omega}$ implies $d G=0$ on $X \backslash \bar{\Omega}$, and then

$$
\int_{X \backslash B_{\varepsilon}(\bar{x})} v d \nu_{\Omega}^{G}=\int_{X \backslash \bar{\Omega}} v d \nu_{\Omega}^{G}=\int_{X \backslash \bar{\Omega}}\langle d G, d v\rangle d \mu=0 .
$$

On the other hand, the harmonicity of $G$ in $\Omega \backslash B_{\varepsilon}(\bar{x})$ implies that if $v \in \operatorname{Lip}_{c}(\Omega \backslash$ $B_{\varepsilon}(\bar{x})$ ), then

$$
\int_{X \backslash B_{\varepsilon}(\bar{x})} v d \nu_{\Omega}^{G}=\int_{\Omega \backslash B_{\varepsilon}(\bar{x})} v d \nu_{\Omega}^{G}=\int_{\Omega \backslash B_{\varepsilon}(\bar{x})}\langle d G, d v\rangle d \mu=0 .
$$

Hence the measure $\nu_{\Omega}^{G}$ is concentrated on $\partial \Omega$. 
Analogously, since $T_{i} G$ is superharmonic in $\Omega$ there exists a positive Radon measure $\nu_{i}^{G} \in N_{0}^{1,2}(\Omega)^{*}$ such that for all $v \in \operatorname{Lip}_{c}(\Omega)$,

$$
\int_{\Omega}\left\langle d T_{i} G, d v\right\rangle d \mu=-\int_{\Omega \backslash B_{r_{i}}(\bar{x})} v d \nu_{i}^{G} .
$$

The measures $\nu_{i}$ are supported in $\bar{B}_{r_{i}}(\bar{x})$; indeed, since $T_{i} G=G$ on $\Omega \backslash \bar{B}_{r_{i}}(\bar{x})$ it is harmonic. Hence, if $v \in \operatorname{Lip}_{c}\left(\Omega \backslash \bar{B}_{r_{i}}(\bar{x})\right)$,

$$
\int_{\Omega} v d \nu_{i}^{G}=\int_{\Omega \backslash \bar{B}_{r_{i}}(\bar{x})} v d \nu_{i}^{G}=\int_{\Omega \backslash \bar{B}_{r_{i}}(\bar{x})}\langle d G, d v\rangle d \mu=0 .
$$

Following the argument of Serrin (see Lemma 1 and Theorem 3 in [22]), there exists $\lambda \in \mathbb{R}$ such that if $v \in \operatorname{Lip}_{c}(\Omega)$ is equal to 1 in a neighborhood of $\bar{x}$, then

$$
\int_{\Omega}\langle d G, d v\rangle d \mu=\lambda
$$

Indeed, if $v_{1}, v_{2} \in \operatorname{Lip}_{c}(\Omega)$ are two functions that are equal to 1 in a neighborhood of $\bar{x}$, the difference $v=v_{1}-v_{2}$ belongs to $\operatorname{Lip}_{c}(\Omega \backslash\{\bar{x}\})$; hence, the harmonicity of $G$ in $\Omega \backslash\{\bar{x}\}$ implies that

$$
\int_{\Omega}\left\langle d G, d v_{1}\right\rangle d \mu-\int_{\Omega}\left\langle d G, d v_{2}\right\rangle d \mu=\int_{\Omega}\langle d G, d v\rangle d \mu=0 .
$$

In particular, if $v \in \operatorname{Lip}_{c}(\Omega)$ is a function such that $v \equiv 1$ on $\bar{B}_{r_{1}}(\bar{x})$, then

$$
\nu_{i}^{G}\left(\bar{B}_{r_{i}}(\bar{x})\right)=\int_{\Omega} v d \nu_{i}^{G}=-\int_{\Omega}\left\langle d T_{i} G, d v\right\rangle d \mu=-\int_{\Omega}\langle d G, d v\rangle d \mu=-\lambda .
$$

This argument implies that $\lambda \in \mathbb{R}$ is negative and the measures $\nu_{i}^{G}$ are equibounded in $\mathscr{M}_{b}(\Omega)$. Thus, up to subsequences, $\nu_{i}^{G}$ converges weakly to $\lambda \delta_{\bar{x}}$.

To summarize, we have proved that the sequence of the measure-valued Laplace operators

$$
\mathscr{D} T_{i} G=\nu_{i}^{G}-\nu_{\Omega}^{G}
$$

admits a convergent subsequence $\mathscr{D} T_{i_{k}} G$, defining the measure-valued Laplace operator

$$
\mathscr{D}_{X} G=\lim _{k \rightarrow \infty} \mathscr{D} T_{i_{k}} G=\lambda \delta_{\bar{x}}-\nu_{\Omega}^{G}
$$

The fact that the limit measure is uniquely determined implies that for any sequence $M_{i} \nearrow \infty$, the measures $\mathscr{D} T_{i} G$ converge and the limit measure is $\lambda \delta_{\bar{x}}-\nu_{\Omega}^{G}$.

Let us show that $\lambda=-1$. Let us consider the set $E=\{x \in \Omega: G(x) \geq 1\}$ and a function $v \in \operatorname{Lip}_{c}(\Omega)$ such that $v=1$ on $E$. Since $\bar{x}$ is an interior point of $E$, we have

$$
\lambda=\lambda v(\bar{x})=-\int_{\Omega \backslash E}\langle d v, d G\rangle d \mu .
$$


On the other hand, the map $f=(G-v) \chi_{\Omega \backslash E}$ belongs to $N_{0}^{1,2}(\Omega \backslash E)$ and then

$$
0=\int_{\Omega}\langle d f, d G\rangle d \mu=\int_{\Omega \backslash E}|d G|^{2} d \mu-\int_{\Omega \backslash E}\langle d v, d G\rangle d \mu .
$$

These properties of $G$ imply that $G$ is the potential of $E$ with respect to $\Omega$, that is,

$$
\int_{\Omega \backslash E}|d G|^{2} d \mu=\operatorname{Cap}_{2}(E, \Omega)=1
$$

We may hence conclude that $\lambda=-1$.

Finally, we point out that the identity

$$
\int_{X}\langle d v, d G\rangle d \mu=\int_{\partial \Omega} v d \nu_{\Omega}^{G}-v(\bar{x})
$$

is valid for functions $v \in N^{1,2}(X)$ that are constant in a neighborhood of $\bar{x}$, but it can be generalized to functions $v \in N^{1,2}(X)$ that are continuous at $\bar{x}$. This is a simple consequence of the limit

$$
\begin{aligned}
\int_{\partial \Omega} v d \nu_{\Omega}^{G}-v(\bar{x}) & =\lim _{i \rightarrow \infty} \int_{\partial \Omega} v d \nu_{\Omega}^{G}-\int_{B_{r_{i}}(\bar{x})} v d \nu_{i}^{G} \\
& =\lim _{i \rightarrow \infty} \int_{X}\left\langle d v, d T_{i} G\right\rangle d \mu=\int_{X}\langle d v, d G\rangle d \mu .
\end{aligned}
$$

Remark 6.2. Let us consider the (first) Heisenberg group $\mathbb{H}$ with the geodesic distance. In this case, the natural differential structure is given by the horizontal bundle and the Laplace operator is just the horizontal Laplace operator. In this setting, we can use all the results of the preceding section and obtain the representation of the measure $\nu^{G}$ in terms of the perimeter measure. Notice that a ball $B_{r}\left(x_{0}\right)$ in $\mathbb{H}$ satisfies a ball condition as in Definition 2.1 of [1] at its boundary except at two points; a finite collection of points is negligible. Hence, if $G=G_{B_{r}\left(x_{0}\right)}^{\bar{x}}$ is the Green function on $B_{r}\left(x_{0}\right)$ with singularity at $\bar{x}$, then whenever $x$ is a boundary point of $B_{r}\left(x_{0}\right)$ satisfying the ball condition,

$$
\Psi(G, x, \varrho):=\sup _{B_{2 \varrho}(x)} G-\sup _{B_{\varrho}(x)} G \leq C \varrho,
$$

where $0<\rho \leq d(x, \bar{x}) / 2$ and $C$ is a positive constant that does not depend on $x, \bar{x}$, or $\rho$. It follows from a covering argument together with (6.1) and Lemma 4.8 in [5] that $\nu_{B_{r}\left(x_{0}\right)}^{G}$ is absolutely continuous with respect to the perimeter measure $\left|D \chi_{B_{r}\left(x_{0}\right)}\right|$. Moreover, there exists a function $\vartheta_{G} \in L^{1}\left(X,\left|D \chi_{B}\right|\right)$ such that $d \nu_{B}^{G}=$ $\vartheta_{G} d\left|D \chi_{B}\right|$. The function $\vartheta_{G}$ comes from the Radon-Nikodym theorem.

We give a characterization of harmonic functions via a mean value type property with respect to boundary measures. 
Theorem 6.3. Let $u \in N^{1,2}(\Omega, \mu)$. Then the following hold:

(1) Let $u$ be harmonic in $\Omega$. Then, for every regular ball $B_{r}\left(x_{0}\right) \subset \Omega$ and $\bar{x} \in$ $B_{r}\left(x_{0}\right)$,

$$
u(\bar{x})=\int_{\partial B_{r}\left(x_{0}\right)} u d \nu_{B_{r}\left(x_{0}\right)}^{G}
$$

(2) If for every regular ball $B_{r}\left(x_{0}\right) \subset \Omega$ and any $\bar{x} \in B_{r}\left(x_{0}\right)$, u satisfies the mean value property (6.2), then $u$ is harmonic in $\Omega$.

An analogous characterization holds true for sub- and superharmonic functions. Let $u \in N^{1,2}(\Omega, \mu)$. Then the following are equivalent:

(3) Let $u$ be subharmonic (superharmonic) in $\Omega$. Then, for every regular ball $B_{r}\left(x_{0}\right) \subset \Omega$ and $\bar{x} \in B$,

$$
u(\bar{x}) \leq \int_{\partial B_{r}\left(x_{0}\right)} u d \nu_{B_{r}\left(x_{0}\right)}^{G}, \quad\left(u(\bar{x}) \geq \int_{\partial B_{r}\left(x_{0}\right)} u d \nu_{B_{r}\left(x_{0}\right)}^{G}\right) ;
$$

(4) If for any regular ball $B_{r}\left(x_{0}\right)$ and any $\bar{x} \in B_{r}\left(x_{0}\right)$

$$
u(\bar{x}) \leq \int_{\partial B_{r}\left(x_{0}\right)} u d \nu_{B_{r}\left(x_{0}\right)}^{G}, \quad\left(u(\bar{x}) \geq \int_{\partial B_{r}\left(x_{0}\right)} u d \nu_{B_{r}\left(x_{0}\right)}^{G}\right),
$$

then $u$ is subharmonic (superharmonic).

Proof. Suppose that $u$ is harmonic. Then $u \in N^{1,2}(\Omega, \mu) \cap L_{\mathrm{loc}}^{\infty}(\Omega)$ and we can apply Theorem 6.1. We obtain, for any regular ball $B_{r}\left(x_{0}\right)$ and $\bar{x} \in B_{r}\left(x_{0}\right)$,

$$
0=\int_{X}\left\langle d u, d G_{B}^{\bar{x}}\right\rangle d \mu=-\int_{X} u d \mathscr{D}_{X} G_{B}^{\bar{x}}=-u(\bar{x})+\int_{\partial B_{r}\left(x_{0}\right)} u d \nu_{B}^{G},
$$

which gives the condition (1).

On the other hand, if $u$ is continuous, if we fix a regular ball $B=B_{r}\left(x_{0}\right)$, we can consider the harmonic function $H_{u}$ generated by $u$ on $B$, that is the solution of the problem

$$
\min \left\{\int_{B}|d v|^{2} d \mu: v-u \in N_{0}^{1,2}(B, \mu)\right\}
$$

Hence $H_{u}$ is harmonic in $B$ and satisfies the mean value property, that is, for any $\bar{x} \in B$,

$$
H_{u}(\bar{x})=\int_{\partial B_{r}\left(x_{0}\right)} H_{u} d \nu_{B}^{G}
$$

The conclusion follows from continuity of $u$ since

$$
\lim _{B \ni x \rightarrow y \in \partial B} H_{u}(x)=u(y)
$$


and then by (6.3), $H_{u}=u$ on $B$. For a general $u \in N^{1,2}(\Omega, \mu)$, we can find a continuous function $u_{\varepsilon}$ such that $u=u_{\varepsilon}$ outside a set of capacity less than $\varepsilon$ and such that $\left\|u-u_{\varepsilon}\right\|_{1,2}<\varepsilon$; then by an approximation argument in Section 6 of [4], we can conclude the assertion.

The same line of reasoning carries out in the case of sub- and superharmonic functions.

Remark 6.4. It was proved in [4] that the harmonic extension of a function $u \in N^{1,2}(\Omega, \mu)$ on a ball $B \subset \Omega$ can be expressed in terms of harmonic measures $\nu_{\bar{x}}$ with singularity at $\bar{x} \in B$; by this we mean that if $\varphi \in C(\partial B)$, then in Theorem 5.1 of [4], its harmonic extension is given by

$$
H_{\varphi}(\bar{x})=\int_{\partial B} \varphi d \nu_{\bar{x}} .
$$

If we move $\bar{x} \in B_{r}\left(x_{0}\right)$, it is possible to see that the measures $\nu_{\bar{x}}$ are mutually equivalent; in particular, if we take $x_{0}$ and $\bar{x} \in B_{r}\left(x_{0}\right) \backslash\left\{x_{0}\right\}$, we have that $\nu_{\bar{x}}$ is absolutely continuous with respect to $\nu_{x_{0}}$ and its density $P(\bar{x}, \cdot)$ is called the Poisson kernel. In other terms, the Poisson kernel is defined as

$$
P(\bar{x}, x)=\frac{d \nu_{\bar{x}}}{d \nu_{x_{0}}}(x) .
$$

In [4], $\nu_{\bar{x}}$ was not explicitly identified. Nevertheless, from the results contained in the previous sections, we are able to identify this measure as the outward normal derivative $\nu_{B}^{G}$ of the Green function.

Example 6.5. In Example 3.5, if we take $\Omega=B_{1}(0)$, the unit ball, then all balls except $B_{1}(0)$ are regular. This is due to the fact that the perimeter of $B_{1}(0)$ has weight 1 , that is $\left|D \chi_{B}\right|=\mathcal{H}^{n-1}\llcorner\partial B$. However, if we consider the optimal sequence $\left(u_{j}\right)_{j}$ defined in $(4.5)$ we have that

$$
\int_{\mathbb{R}^{n}}\left|\nabla u_{j}\right| d \mu \rightarrow 2 \mathcal{H}^{n-1}\left(\partial B_{1}(0)\right)=2\left|D \chi_{B_{1}(0)}\right|\left(\mathbb{R}^{n}\right) .
$$

Nevertheless, the measure $\nu_{B_{1}(0)}^{G}$ can still be characterized as a perimeter measure, but with

$$
d \nu_{B_{1}(0)}^{G}=2\left(\nabla G \cdot \nu_{B_{1}(0)}\right) d \mathcal{H}^{n-1}\left\llcorner\partial B_{1}(0)=2\left(\nabla G \cdot \nu_{B_{1}(0)}\right) d\left|D \chi_{B_{1}(0)}\right| .\right.
$$

On the other hand, if we take any other ball $B \subset B_{1}(0)$, it is regular and in this case $\mathcal{H}^{n-1}\left(\partial B \cap \partial B_{1}(0)\right)=0$. Note also that if $\mathcal{H}^{n-1}\left(B \cap \partial B_{1}(0)\right)>0$, then since the Green function is harmonic in $B$ except the singular point $\bar{x}$, we have that $\nabla G \cdot \nu_{B_{1}(0)}=0$ and then

$$
d \nu_{B}^{G}=\left(1+\chi_{B_{1}(0)}\right)\left(\nabla G \cdot \nu_{B}\right) d \mathcal{H}^{n-1}\left\llcorner\partial B=\left(\nabla G \cdot \nu_{B}\right) d\left|D \chi_{B}\right|\right.
$$

On the other hand, if we take $\Omega=\mathbb{R}^{n} \backslash \overline{B_{1}}(0)$, then every ball is regular. This is due to the fact that in this paper regularity is a notion of inner regularity. If one changes the notion to outer regularity or to two-sided regularity, then things change. 


\section{References}

[1] Aikawa, H., Kilpeläinen, T., Shanmugalingam, N. and Zhong, X.: Boundary Harnack principle for $p$-harmonic functions in smooth Euclidean domains. Potential Anal. 26 (2007), no. 3, 281-301.

[2] Ambrosio, L.: Fine properties of sets of finite perimeter in doubling metric measure spaces. Set-Valued Anal. 10 (2002), no. 2-3, 111-128.

[3] Ambrosio, L., Miranda JR., M. and Pallara, D.: Special functions of bounded variation in doubling metric measure spaces. In Calculus of variations: topics from the mathematical heritage of E. De Giorgi, 1-45. Quad. Mat. 14, Dept. Math., Seconda Univ. Napoli, Caserta, 2004.

[4] Björn, A., Buörn, J. And Shanmugalingam, N.: The Dirichlet problem for $p$ harmonic functions on metric spaces. J. Reine Angew. Math. 556 (2003), 173-203.

[5] Buörn, J., Macmanus, P. and Shanmugalingam, N.: Fat sets and pointwise boundary estimates for $p$-harmonic functions in metric spaces. J. Anal. Math. $\mathbf{8 5}$ (2001), 339-369.

[6] Camfield, C.: Comparison of BV norms in weighted Euclidean spaces and metric measure spaces. Ph.D. Thesis, University of Cincinnati, 2008.

[7] Cheeger, J.: Differentiability of Lipschitz functions on metric measure spaces. Geom. Funct. Anal. 9 (1999), no. 3, 428-517.

[8] Chen, C. Q., Torres, M. And Ziemer, W. P.: Measure-theoretic analysis and nonlinear conservation laws. Pure Appl. Math. Q. 3 (2007), no. 3, 841-879.

[9] Danielli, D., Garofalo, N. and Marola, N.: Local behavior of $p$-harmonic Green's functions in metric spaces. Potential Anal. 32 (2010), no. 4, 343-362.

[10] Franchi, B., Hajeasz, P. and Koskela, P.: Definitions of Sobolev classes on metric spaces. Ann. Inst. Fourier (Grenoble) 49 (1999), no. 6, 1903-1924.

[11] Fukushima, M., Oshima, Y. And Takeda, M.: Dirichlet forms and symmetric Markov processes. De Gruyter Studies in Mathematics 19, Walter de Gruyter, Berlin, 1994.

[12] HajŁasz, P.: Sobolev spaces on metric-measure spaces. In Heat kernels and analysis on manifolds, graphs, and metric spaces (Paris, 2002), 173-218. Contemp. Math. 338, Amer. Math. Soc., Providence, RI, 2003.

[13] Hajeasz, P. and Koskela, P.: Sobolev met Poincaré. Mem. Amer. Math. Soc. 145, (2000), no. 688, 1-101.

[14] Holopainen, I.: Nonlinear potential theory and quasiregular mappings on Riemannian manifolds. Ann. Acad. Sci. Fenn. Ser. A I Math. Dissertationes 74 (1990), $1-45$.

[15] Holopainen, I. And Shanmugalingam, N.: Singular functions on metric measure spaces. Collect. Math. 53 (2002), no. 3, 313-332.

[16] Kallunki, S. and Shanmugalingam, N.: Modulus and continuous capacity. Ann. Acad. Sci. Fenn. Math. 26 (2001), no. 2, 455-464.

[17] Keith, S.: Modulus and the Poincaré inequality on metric measure spaces. Math. Z. 245 (2003), no. 2, 255-292.

[18] Kinnunen, J. And Shanmugalingam, N.: Regularity of quasi-minimizers on metric spaces. Manuscripta Math. 105 (2001), no. 3, 401-423. 
[19] Koskela, P., Rajala, K. and Shanmugalingam, N.: Lipschitz continuity of Cheeger-harmonic functions in metric measure spaces. J. Funct. Anal. 202 (2003), no. $1,147-173$.

[20] Mikkonen, P.: On the Wolff potential and quasilinear elliptic equations involving measures. Ann. Acad. Sci. Fenn. Math. Diss. 104 (1996), 1-71.

[21] Miranda JR., M.: Functions of bounded variation on "good" metric spaces. J. Math. Pures Appl. (9) 82 (2003), no. 8, 975-1004.

[22] Serrin, J.: Isolated singularities of solutions of quasi-linear equations. Acta Math. 113 (1965), 219-240.

[23] Shanmugalingam, N.: Newtonian spaces: an extension of Sobolev spaces to metric measure spaces. Rev. Mat. Iberoamericana 16 (2000), no. 2, 243-279.

[24] Shanmugalingam, N.: Harmonic functions on metric spaces. Illinois J. Math. 45 (2001), no. 3, 1021-1050.

[25] Sturm, K. T.: Analysis on local Dirichlet spaces. III. The parabolic Harnack inequality. J. Math. Pures Appl. (9) 75 (1996), no. 3, 273-297.

[26] Thompson, A. A. And Thompson, A. C.: The divergence theorem and the Laplacian in Minkowski space. Geom. Dedicata 63 (1996), no. 2, 159-170.

[27] Ziemer, W. P.: The Gauss-Green theorem for weakly differentiable vector fields. In Singularities in PDE and the calculus of variations, 233-267. CRM Proc. Lecture Notes 44, Amer. Math. Soc., Providence, RI, 2008.

Received April 16, 2013.

Niko Marola: Department of Mathematics and Statistics, University of Helsinki, P.O. Box 68 (Gustaf Hällströmin katu 2b), 00014 University of Helsinki, Finland; and Aalto University, Department of Mathematics and Systems Analysis, P.O. Box 11100, 00076 Aalto, Finland.

E-mail: niko.marola@helsinki.fi

Michele Miranda JR.: Department of Mathematics and Computer Science, University of Ferrara, via Machiavelli 35, 44121 Ferrara, Italy.

E-mail: michele.miranda@unife.it

Nageswari Shanmugalingam: Department of Mathematical Sciences, University of Cincinnati, P.O. Box 210025, Cincinnati, OH 45221-0025, USA.

E-mail: shanmun@uc.edu

Nageswari Shanmugalingam was partially supported by NSF grant DMS-0243355, Simons Foundation grant no. 200474, and NSF grant DMS-1200915. 